\title{
Difeomorfismos que Preservam Órbitas de Campos de Vetores Lineares Hiperbólicos
}

\author{
LuCiana de FÁtima Martins ${ }^{1}$ \\ Orientador: \\ Prof. Dr. José Luis Arraut Vergara
}

ـ voo rtação apresentada ao Instituto de Ciências Matemáticas e de Computação da Universidade de São Paulo, como parte dos requisitos para a obtenção do título de "Mestre em Ciências - Área: Matemática".

USP - Sāo Carlos

Janeiro de 1999

\footnotetext{
${ }^{1}$ Este trabalho teve suporte financeiro inicial da CAPES e final da FAPESP.
} 


\section{Agradecimentos}

Não pretendo me estender muito nesta parte pois, se eu quiser ser justa nos agradecimentos, talvez gaste mais tempo do que levei para escrever esta dissertação. Contudo não posso deixar de mencionar alguns nomes que me ajudaram a escrever as páginas de mais esta vitória.

À Deus, pela presença constante em minha vida.

Como não poderia deixar de ser, quero agradecer à minha familia que sempre esteve presente, mesmo distante, principalmente: meu pai Milton e minha mãe Iracy.

A meu orientador, Prof. Arraut, pela dedicação e paciência que teve comigo ao longo deste ano que passou.

$\dot{A} D$. Neide e ao S. Sidney, que me ajudaram demais, principalmente nos periodos de mudança.

Aos professores do departamento de Matemática do ICMC, principalmente à Profa. Maria Ap. Soares Ruas (Cidinha), Ozíride M. Neto (Dide), Prof. Plácido, Prof. Biasi e Maria do Carmo, com os quais muito aprendi.

As professoras da UNESP de Rio Claro: Nativi, Rosa, Cristina, Solange e Alice, pelas quais guardo um enorme carinho; em especial aos professores Marcelo José Saia (meu orientador de iniciação científica) e Prof. Loibel, por terem sido grandes amigos.

Ainda de Rio Claro, não poderia deixar de agradecer ao Sr. Romildo Zanelatto e sua esposa D. Darcy, pelo carinho que tiveram comigo.

Ao Cavassini (Zé) e ao Zardetto, meus queridos amigos.

Algumas amizades mais recentes, porém de importância indescritivel, também não poderiam faltar: Marcão, Regilene e Maria do Carmo, sempre dispostos a me ajudar quando preciso; meus colegas de turma: José, Eliane, Márcio (sua presença foi fundamental, obrigada amigo!), Maria Alice e Vera (duas irmãzinhas que ganhei neste último ano).

Gostaria de terminar estes agradecimentos citando o Fernando, meu noivo $e$ amigo, que sabe muito bem de sua importância na realização desta dissertação, a qual era o nosso objetivo quando saímos de Assis e agora se torma realidade. Devo isso a você!

Para terminar, enfim, agradeço às secretárias da pós-graduação Marlita, Beth $e$ Laura, pela eficiência e simpatia com que tratam nossos assuntos. 
Ao Fernando

"Sonho que se sonha só, é só um sonho que se sonha só, mas sonho que se sonha junto, é realidade!" 


\section{Resumo}

Sejam $X$ um campo de vetores linear hiperbólico em $\mathbb{R}^{n}$, de índice $n$ (ou 0 ), $U$ um aberto de $\mathbb{R}^{n}$ contendo a origem e $f: U \rightarrow \mathbb{R}^{n}$ um $C^{r}$-difeomorfismo local, $r \geq 1$, que manda órbitas de $X$ em $U$ em órbitas de $X$ em $\mathbb{R}^{n}$, preservando a orientação. Escrevendo $U_{0}=U-\{0\}$, seja $\frac{U_{0}}{X}$ a variedade diferenciável obtida ao identificar cada órbita de $X \mathrm{em} U$ a um ponto. Neste trabalho estudamos a aplicação induzida por $f, \bar{f}: \frac{U_{0}}{X} \rightarrow \frac{\mathbb{R}_{0}^{n}}{X}$. Fazemos ainda um estudo mais detalhado para o caso em que $n=2$. Esta dissertação é baseada no artigo [1] de C. Camacho e A. Lins Neto. 


\section{Abstract}

Let $X$ be a hyperbolic linear vector field on $\mathbb{R}^{n}$, with index $n$ (or 0 ), $U$ be an open set in $\mathbb{R}^{n}$ containing the origin and $f: U \rightarrow \mathbb{R}^{n}$ a $C^{r}$-diffeomorphism, $r \geq 1$, such that $f$ maps orbits of $X$ in $U$ to orbits of $X$ in $\mathbb{R}^{n}$ and preserves the orientation. Denote $U_{0}=U-\{0\}$ and let $\frac{U_{0}}{X}$ be the differentiable manifold obtained by identifying each orbit of $X$ in $U$ to a point. In this work we study the map induced by $f, \bar{f}: \frac{U_{0}}{X} \rightarrow \frac{\mathbb{R}_{0}^{n}}{X}$. We also study with more detail the case $n=2$. This dissertation is based on the paper [1] by C. Camacho and A. Lins Neto. 


\section{Sumário}

$\begin{array}{ll}\text { Introduçāo } & 1\end{array}$

1 Conceitos básicos e notaçōes . 3

1.1 Campos de vetores .................... 3

1.1.1 Campos lineares . . . . . . . . . . . . . . . 4

1.1.2 Equivalência de campos de vetores ............ 7

1.2 Difeomorfismos .................... 8

2 Principais resultados 11

2.1 Considerações iniciais e lemas preliminares . . . . . . . . . . . 12

2.2 Prova do Teorema $2.1 \ldots \ldots \ldots$

2.3 Prova do Teorema $2.2 \ldots \ldots . \ldots \ldots$. . . . . . . . . 42

3 Dinâmica da aplicaçāo induzida para $X \in \mathcal{H}\left(\mathbb{R}^{2}\right) \quad 48$

3.1 Autovalores complexos conjugados . . . . . . . . . . . . . 49

3.2 Autovalores reais . . . . . . . . . . . . . . . . . 49

3.2.1 Autovalores distintos e de mesmo sinal . . . . . . . . 55

3.2.2 Autovalores de sinais opostos ............ 56

3.2.3 Autovalores iguais ................ 58

3.3 Observação ........................ 66

$\begin{array}{ll}\text { Referências Bibliográficas } & 67\end{array}$ 


\section{Introdução}

Sejam $X$ um campo de vetores linear hiperbólico em $\mathbb{R}^{n}$ e $f: U \rightarrow \mathbb{R}^{n}$ um $C^{r}$-difeomorfismo local, $r \geq 1$, com $U$ aberto de $\mathbb{R}^{n}, 0 \in U$, tal que $f$ aplica órbitas de $X$ em $U$ em órbitas de $X$ em $\mathbb{R}^{n}$. Definindo $U_{0}=U-\{0\}$, seja $\frac{U_{0}}{X}$ o espaço topológico com a topologia quociente obtido ao identificar cada órbita de $X$ em $U$ a um ponto. $\frac{U_{0}}{X}$ é de fato uma variedade diferenciável $C^{\infty}$ de dimensão $n-1$, não necessariamente Hausdorff, que pode ser realizada via um subconjunto do $\mathbb{R}^{n}$. Por exemplo, se $X$ for um campo de vetores linear hiperbólico em $\mathbb{R}^{2}$ cujos autovalores satisfazem $\alpha_{2}<\alpha_{1}<0$ então $\frac{U_{0}}{X}$ pode ser realizada como sendo a $S^{1}$ (veja figura abaixo). De fato, cada órbita de $X$ cruza transversalmente $S^{1}$ em um único ponto.

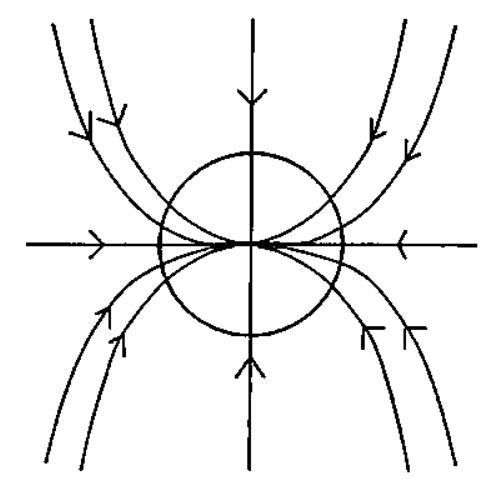

Figura 1: As órbitas do campo $X$, onde seus autovalores $\alpha_{1}$ e $\alpha_{2}$ são tais que $\alpha_{2}<\alpha_{1}<0$ e $S^{1}$.

Se $f: U \rightarrow \mathbb{R}^{n}$ é como acima, então $f$ induz um difeomorfismo de classe $C^{r}$ $\bar{f}: \frac{U_{0}}{X} \rightarrow \frac{\mathbb{R}_{0}^{n}}{X}$ de tal maneira que o diagrama abaixo comuta: 


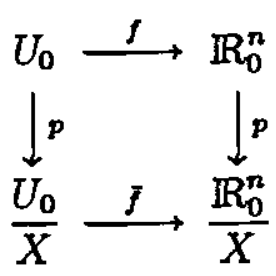

onde $p$ é a aplicação quociente.

Tais difeomorfismos são estudados nos artigos [1] e [2], o primeiro de C. Camacho e A. Lins Neto e o último de J. L. Arraut. O conteúdo desta dissertação tem como base estes dois artigos.

No Capítulo 1 introduzimos notações, definições e resultados básicos sobre campos de vetores e difeomorfimos, os quais facilitarão a leitura deste trabalho.

No Capítulo 2 fazemos uma exposição, em detalhes, dos teoremas 1 e 2 de [1], que aqui aparecem como Teorema 2.1 e Teorema 2.2, respectivamente. Estes dois teoremas proporcionam uma boa informação sobre $\bar{f}$ no caso em que os autovalores de $X$ são de multiplicidade 1 e de mesmo sinal. Para facilitar a compreensão destes teoremas é feita primeiro, aqui, uma demonstração no caso particular $n=2$. Para terminar o capítulo apresentamos a demonstração do caso geral.

No Capítulo 3 fizemos um estudo mais completo de $\vec{f}$ para o caso $n=2$. Aqui aproveita-se o Lema 3.2 de [2], que calcula a derivada de $\bar{f}$ nos pontos periódicos e os resultados do capítulo anterior. O material deste capítulo pode ser sintetizado pelo seguinte teorema:

Teorema 0.1 Sejam $X$ um campo de vetores linear hiperbólico em $\mathbb{R}^{2}$ ef $U \rightarrow \mathbb{R}^{2}$ um difeomorfismo local que aplica órbitas de $X$ em $U$ em órbitas de $X$ em $\mathbb{R}^{2}$, preservando a orientação, onde U é uma vizinhança da origem em $\mathbb{R}^{2}$. Sejam $\alpha$ e $\beta$ os autovalores de $X$ e a e b os correspondentes de $D f(0)$. Então $\vec{f}$ tem um número finito de pontos periódicos, todos eles hiperbólicos, se e somente se $\alpha, \beta \in \mathbb{R} e$ $\alpha \ln |b| \neq \beta \ln |a|$. 


\section{Capítulo 1}

\section{Conceitos básicos e notações}

Este capítulo contém as definições e os resultados básicos envolvendo campos de vetores e difeomorfismos, estabelecendo a linguagem necessária à compreensão dos capítulos posteriores. Para mantermos a simplicidade na notação, tais resultados serão enunciados dentro do contexto em que estaremos trabalhando e, sendo admitidos conhecidos, não nos preocuparemos com maiores detalhes. Nossa principal referência é o livro [3] porém contamos também como referência de apoio os livros [4], [5] e [6].

\subsection{Campos de vetores}

Salvo menção explícita em contrário, $U$ representará um subconjunto aberto do espaço euclidiano $n$-dimensional real $\mathbb{R}^{n}$.

Definiçāo $1.1 U m$ campo de vetores de classe $C^{r}$ em $U, r \geq 1$, é uma aplicação de classe $C^{r} X: U \rightarrow \mathbb{R}^{n}$ que a cada ponto $p \in U$ associa um vetor $X(p) \in \mathbb{R}^{n}$.

Uma curva integral de $X$, por ụm ponto $p \in U$, é uma aplicação diferenciável $\alpha: I \rightarrow U\left(I\right.$ intervalo da reta contendo a origem) tal que $\alpha(0)=p$ e $\alpha^{\prime}(t)=X(\alpha(t))$, para todo $t \in I$. Assim, $\alpha$ é uma solução da equação diferencial $\frac{d x}{d t}=X(x)$, com condição inicial $x(0)=p$. A imagem de uma curva integral é chamada de órbita 
ou trajetória do campo $X$.

O teorema abaixo nos garante a existência e unicidade da curva integral de um campo de vetores com condição inicial $p \in U$ (ou da solução da equação diferencial associada ao campo).

Teorema 1.1 ([3], pag 4)(Existência e Unicidade) Sejam X um campo de classe $C^{r}, r \geq 1$ e $p \in U$. Existe uma curva integral de $X, \alpha: I \rightarrow U \operatorname{com} \alpha(0)=p$. Se $\beta: J \rightarrow U$ é outra curva integral de $X, \operatorname{com} \beta(0)=p$, então $\alpha(t)=\beta(t)$, para todo $t \in I \cap J$.

Denotaremos por $\mathfrak{X}^{r}(U)$ o conjunto dos campos de vetores de classe $C^{r}$ definidos em $U, \operatorname{com} r \geq 1$.

\subsubsection{Campos lineares}

Em nosso estudo estaremos considerando campos de vetores lineares. Quando um campo $X$ for linear denotaremos, com um abuso de notação, a matriz do campo também por $X$.

Definiçāo 1.2 Seja $X \in \mathfrak{X}^{r}\left(\mathbb{R}^{n}\right), r \geq 1$. Um fluxo global de $X$ em $\mathbb{R}^{n}$ é uma aplicação $\varphi: \mathbb{R} \times \mathbb{R}^{n} \rightarrow \mathbb{R}^{n}$ tal que, para cada $p \in \mathbb{R}^{n}$, a aplicação $\varphi_{p}: \mathbb{R} \rightarrow \mathbb{R}^{n}$ definida por $\varphi_{p}(t)=\varphi(t, p)$ é uma curva integral passando por $p$; isto é, $\varphi(0, p)=p$ $e \frac{\partial \varphi}{\partial t}(t, p)=X(\varphi(t, p))$, para todo $(t, p) \in \mathbb{R} \times \mathbb{R}^{n}$.

O teorema abaixo nos garante a existência (e unicidade) de um fluxo global para um campo linear.

Teorema 1.2 ([3], pag 47) Seja $X$ um campo linear em $\mathbb{R}^{n}$. Então a aplicação $\varphi: \mathbb{R} \times \mathbb{R}^{n} \rightarrow \mathbb{R}^{n}$ definida por $\varphi(t, x)=e^{t X} x$ é o fluxo global do campo $X$.

Seja $X$ um campo linear. A órbita de $X$ por $p \in \mathbb{R}^{n}$ é o conjunto $\mathcal{O}(p)=\left\{X_{t}(p) ; \quad t \in \mathbb{R}\right\}$, onde $X_{t}(p)=\varphi(t, p)$, com $\varphi$ o fluxo de $X$ por $p$ no 
instante $t$.

Segue do Teorema 1.2 que duas órbitas de $X$ coincidem ou são disjuntas. Temos assim que $\mathbb{I R}^{n}$ fica decomposto numa união disjunta de curvas diferenciáveis. $O$ espaço $\mathbb{R}^{n}$ munido da decomposição em órbitas de $X$ é chamado de retrato de fase de $X$.

O resultado seguinte possibilita escrever a matriz de um campo linear de uma maneira bem simples.

Teorema 1.3 ([3], pag 48) (Forma Canônica de Jordan Real) Se X é um campo linear em $\mathbb{R}^{n}$, existe uma base de $\mathbb{R}^{n}$ na qual a matriz de $X$ tem a forma

$$
\left(\begin{array}{cccccc}
A_{1} & & & & 0 & \\
& \ddots & & & & \\
& & A_{r} & & & \\
& & & B_{1} & & \\
& & & & \ddots & \\
& & & & & B_{s}
\end{array}\right) \text {, }
$$

onde

$$
\begin{aligned}
& A_{i}=\left(\begin{array}{ccccc}
\lambda_{i} & 1 & & 0 & \\
& \lambda_{i} & 1 & & \\
& & \ddots & \ddots & \\
& & & \lambda_{i} & 1 \\
& 0 & & & \lambda_{i}
\end{array}\right) i=1, \ldots, r \quad \lambda_{i} \in \mathbb{R} \\
& B_{j}=\left(\begin{array}{cccc}
C_{j} & I & & 0 \\
& \ddots & \ddots & \\
& & C_{j} & I \\
0 & & & C_{j}
\end{array}\right),
\end{aligned}
$$




$$
C_{j}=\left(\begin{array}{rr}
\alpha_{j} & \beta_{j} \\
-\beta_{j} & \alpha_{j}
\end{array}\right) \alpha_{j}, \beta_{j} \in \mathbb{R} \quad \text { e } I=\left(\begin{array}{cc}
1 & 0 \\
0 & 1
\end{array}\right)
$$

As submatrizes $A_{1}, \ldots, A_{r}$ e $B_{1}, \ldots, B_{s}$ são univocamente determinadas a menos da ordem.

Exemplo 1.1 Se $X$ é um campo linear em $\mathbb{R}^{2}$ então a matriz de $X$ possui uma das formas abaixo, exceto por uma mudança de base:

$$
\left(\begin{array}{cc}
\lambda_{1} & 0 \\
0 & \lambda_{2}
\end{array}\right) ;\left(\begin{array}{cc}
\lambda & 1 \\
0 & \lambda
\end{array}\right) ;\left(\begin{array}{rr}
\alpha & \beta \\
-\beta & \alpha
\end{array}\right),
$$

sendo as entradas das matrizes números reais.

Seja $X$ um campo de vetores linear em $\mathbb{R}^{n}$. Ao conjunto de autovalores de $X$ denominaremos espectro de $X$.

Definiçāo 1.3 Um campo linear $X$ é dito hiperbólico se o espectro de $X$ é disjunto do eixo imaginário. $O$ número de autovalores de $X$, contando suas multiplicidades, com parte real negativa é denominado índice de $X$.

O conjunto dos campos de vetores lineares hiperbólicos em $\mathbb{R}^{n}$ será denotado por $\mathcal{H}\left(\mathbb{R}^{n}\right)$.

Proposiçāo 1.1 ([3], pag 51)Se $X \in \mathcal{H}\left(\mathbb{R}^{n}\right)$, então existe uma única decomposição de $\mathbb{R}^{n}$ em soma direta $\mathbb{R}^{n}=E^{s} \oplus E^{u}$, onde $E^{s}$ e $E^{u}$ são subespaços invariantes por $X$ e pelo fluxo associado a $X$ tais que os autovalores de $X^{s}=\left.X\right|_{E^{*}}$ têm parte real negativa e os autovalores de $X^{u}=\left.X\right|_{E^{u}}$ têm parte real positiva.

Proposiçāo 1.2 ([3], pag 54) Se $X \in \mathcal{H}\left(\mathbb{R}^{n}\right)$ e $\mathbb{R}^{n}=E^{s} \oplus E^{u}$ é a decomposição da proposição acima, então $X_{t}(x)$ converge para a origem se $x \in E^{s}$ et $\rightarrow+\infty e$ $X_{t}(x)$ converge para a origem se $x \in E^{u}$ et $\rightarrow-\infty$. 
Lema 1.1 ([3], pag 56) Seja $X$ um campo linear de $\mathbb{R}^{n}$ de índice $n$. Existe uma norma $\|\cdot\|$ em $\mathbb{R}^{n}$ tal que, se $S^{n-1}=\left\{v \in \mathbb{R}^{n} ;\|v\|=1\right\}$, então $T S_{x}^{n-1}$ e $X(x)$ geram o espaço $\mathbb{R}^{n}$ para todo $x \in S^{n-1}$, onde $T S_{x}^{n-1}$ é o espaço tangente a $S^{n-1}$ em $x$.

Observaçāo 1.1 Do Lema 1.1 e da proposição 1.2 segue que se $x \in \mathbb{R}^{n}-\{0\}$, então a órbita de $x$ interseta $S^{n-1}$ em um único ponto.

Definiçāo 1.4 Seja $X$ um campo linear em $\mathbb{R}^{n}$. Dizemos que os autovalores de $X,\left\{\mu_{1}, \ldots, \mu_{n}\right\}$, satisfazem as condições de nāo-ressonância se, para qualquer sequiência $\left(m_{1}, \ldots, m_{n}\right)$ de inteiros não-negativos com $\sum_{j=1}^{n} m_{j} \geq 2$, tem-se que $\mu_{i} \neq \sum_{j=1}^{n} m_{j} \mu_{j}$, para todo $i=1, \ldots, n$

\subsubsection{Equivalência de campos de vetores}

Definiçāo 1.5 Sejam $X, Y \in \mathfrak{X}^{+}\left(\mathbb{R}^{n}\right)$ e $f: \mathbb{R}^{n} \rightarrow \mathbb{R}^{n}$ um homeomorfismo. Dizemos que $f$ preserva órbitas se $f$ leva órbitas de $X$ em órbitas de $Y$, preservando $a$ orientação. Mais precisamente, se $p \in \mathbb{R}^{n}$ então existe $\sigma: \mathbb{R} \rightarrow \mathbb{R}$, um homeomorfismo crescente, tal que $f\left(X_{t}(p)\right)=Y_{\sigma(t)}(f(p))$. Se $\sigma(t)=t$ então dizemos que $f$ também preserva o parâmetro.

Consideremos a seguinte relação de equivalência em $\mathfrak{X}^{r}\left(\mathbb{R}^{n}\right)$, a qual permite comparar os retratos de fase de dois campos de vetores: dois campos $X, Y \in \mathfrak{X}^{+}\left(\mathbb{R}^{n}\right)$ são topologicamente equivalentes se existe um homeomorfismo $h: \mathbb{R}^{n} \rightarrow \mathbb{R}^{n}$ que preserva órbitas. Neste caso $h$ é chamada uma equivalência topológica entre $X$ e $Y$. Se o homeomorfismo acima também preservar o parâmetro então $h$ é dito ser uma conjugação entre os campos $X$ e $Y$.

Proposiçāo 1.3 ([3], pag 57) Sejam X e Y campos lineares hiperbólicos. Então $X$ e $Y$ säo topologicamente equivalentes se e somente se têm o mesmo índice. 
Para o caso em que os campos lineares hiperbólicos $X$ e $Y$ são tais que $X=$ $P^{-1} Y P$, onde $P$ é uma matriz inversível, o seguinte resultado garante a existência de um difeomorfismo que conjuga os campos.

Teorema 1.4 Dois campos lineares hiperbólicos $X$ e $Y$ são diferenciavelmente conjugados (isto $\ell$, existe um difeomorfismo $h: \mathbb{R}^{n} \rightarrow \mathbb{R}^{n}$ satisfazendo $\left.h\left(X_{t}(p)\right)=Y_{t}(h(p))\right)$ se e somente se eles satisfazem $X=P^{-1} Y P$ para alguma matriz inversivel $P$.

\section{Demonstraçāo:}

Segue das Proposiçōes 5 e 8 de [4], páginas 71 e 72.

\subsection{Difeomorfismos}

Análogo ao estudo feito para campos de vetores, existe um estudo paralelo para difeomorfismos que tem sido da maior relevância para a compreensão dos espaços de fase dos campos de vetores. Iniciaremos este parágrafo introduzindo o conceito de variedade diferenciável, definindo-a como um subconjunto de $\mathbb{R}^{n}$. Observamos que existe um conceito abstrato de variedade que, a priori, seria mais geral do que aquele que apresentaremos aqui. Porém, para o contexto em que trabalharemos, a definição apresentada simplificará a exposição.

Seja $M$ um subconjunto do espaço euclidiano $\mathbb{R}^{k}$. Consideremos em $M$ a topologia induzida, isto é, $A \subset M$ é aberto se existe um aberto $A^{\prime} \subset \mathbb{R}^{k}$ tal que $A=A^{\prime} \cap M$. Dizemos que $M \subset \mathbb{R}^{k}$ é uma variedade diferenciável de dimensão $m$ se, para cada ponto $p \in M$, existem uma vizinhança $U \subset M$ de $p$ e um homeomorfismo $\mathbf{x}: U \rightarrow U_{0}, U_{0}$ um aberto de $\mathbb{R}^{m}$, tais que o homeomorfismo inverso $\mathbf{x}^{-1}: U_{0} \rightarrow U \subset \mathbb{R}^{k}$ é uma imersão de classe $C^{\infty}$; isto é, para cada $u \in U_{0}$, a derivada $d \mathbf{x}^{-1}(u): \mathbb{R}^{m} \rightarrow \mathbb{R}^{k}$ é injetora de classe $C^{\infty}$. Dizemos nesse caso que $(U, \mathbf{x})$ é uma carta local em torno de $p$ e $U$ é uma vizinhança coordenada de $p$. 
Se o homeomorfismo $\mathbf{x}^{-1}$ acima é de classe $C^{r}$, dizemos que $M$ é uma variedade de classe $C^{r}$.

Usamos o par $(U, \mathbf{x})$ para dar significado à diferenciabilidade em $U$. Assumindo, por simplicidade, $f: U \rightarrow U$, então $f$ induz uma aplicação $\tilde{f}=\operatorname{xof}^{\circ} \circ \mathbf{x}^{-1}: U_{0} \rightarrow U_{0}$, conforme diagrama abaixo:

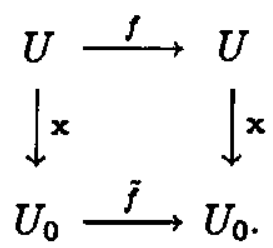

Dizemos que $f$ é uma aplicação de classe $C^{k}$ em $U$ se $\tilde{f}$ for uma aplicação de classe $C^{k}$ em $U_{0}$. Seja $D i f^{r}(M)$ o conjunto dos difeomorfismos de classe $C^{r}$ de $M$ em $M \subset \mathbb{R}^{s}$, onde $M$ é uma variedade compacta de dimensão $m$ com a topologia $C^{r}$, isto é, induzida pela norma \|\|$_{r}$, dada como segue: tome em $M$ uma cobertura finita por abertos $V_{1}, \ldots, V_{k}$ tal que cada $V_{i}$ esteja contido no domínio de uma carta local $\left(\mathrm{x}_{i}, U_{i}\right)$ com $\mathrm{x}_{i}\left(U_{i}\right)=B(2)$ e $\mathrm{x}_{i}\left(V_{i}\right)=B(1)$, onde $B(1)$ e $B(2)$ são as bolas de raio 1 e 2 e centro na origem de $\mathbb{R}^{m}$. Para cada $f \in D_{i} f^{r}(M)$ denotamos por $f^{i}=f \circ \mathbf{x}_{i}^{-1}: B(2) \rightarrow \mathbb{R}^{s}$. Então

$$
\|f\|_{r}=\max _{i} \sup _{u \in B(1)}\left\{\left\|f^{i}(u)\right\|,\left\|d f^{i}(u)\right\|, \cdots,\left\|d^{r} f^{i}(u)\right\|\right\} .
$$

Para majores detalhes ver [3], pag 22.

Sejam $p \in M$ e $f \in D i f^{r}(M)$. A órbita de $p$ é o conjunto $\mathcal{O}(p)=\left\{f^{n}(p) ; n \in\right.$ $\mathbb{Z}\}$. Dizemos que $p$ é um ponto periódico de $f$ se existe um inteiro $n>0$ tal que $f^{n}(p)=p$. O menor inteiro $n$ tal que isto acontece é chamado de período de $p$. Se $p$ é periódico de período 1 , ou seja, $f(p)=p$, dizemos que $p$ é um ponto fixo de $f$.

Definiçāo 1.6 Seja $p \in M$ um ponto periódico de $f \in D_{i f}(M)$ de período $m$. Dizemos que pé um ponto periódico hiperbólico de f se $D f_{p}^{m}: T M_{p} \rightarrow T M_{p}$ não tem autovalor de norma 1. 
Teorema 1.5 ([3], pag. 65) O conjunto dos difeomorfismos cujos pontos fixos são todos hiperbólicos é aberto e denso em Difr $(M)$.

Definiçäo 1.7 Seja $f \in D_{i f}(M)$. Um ponto $p \in M$ é nāo-errante para $f$ se para qualquer vizinhança $U$ de $p$ e qualquer inteiro $n_{0}>0$ existe um inteiro $n$ tal que $|n|>n_{0}$ e $f^{n}(U) \cap U \neq \emptyset$.

Denotamos por $\Omega(f)$ o conjunto dos pontos não-errantes de $f$.

Observaçāo 1.2 Algumas propriedades importantes de $\Omega(f)$ são:

1. $\Omega(f)$ é fechado e também invariante, isto é, se $p \in \Omega(f)$ então $\mathcal{O}(p) \subset \Omega(f)$;

2. todo ponto fixo ou periódico de $f$. pertence a $\Omega(f)$.

A próxima definição expressa a equivalência entre as estruturas de órbitas de. dois difeomorfismos.

Definiçāo $1.8 \operatorname{Sejam~} f, g \in \operatorname{Dif}^{r}(M)$. Uma conjugaçāo topológica entre $f e$ $g$ é um homeomorfismo $h: M \rightarrow M$ tal que $h \circ f=g \circ h$. Dizemos que $f$ é estruturalmente estável se existe uma vizinhança $\mathcal{V}$ de $f$ em $\operatorname{Difr}^{r}(M)$ tal que qualquer $g \in \mathcal{V}$ é topologicamente conjugado a f.

Se $h$ é como na definição acima então $h$ leva cada órbita de $f$ sobre uma órbita de $g$, preservando o parâmetro $m$, isto é,

$$
f^{m}(x) \stackrel{h}{\longrightarrow} g^{m}(h(x)), \text { paracada } m \in \mathbb{Z} .
$$

Enunciaremos agora um resultado que caracteriza a estabilidade estrutural de difeomorfismos definidos sobre $S^{1}$.

Teorema 1.6 ([6], pg 132) Um difeomorfismo $f \in D_{i f^{1}}\left(S^{1}\right)$ é estruturalmente estável se e somente se seu conjunto de pontos não-errantes, $\Omega(f)$, consiste de um numero finito de pontos fixos e periódicos, todos hiperbólicos. Além disso, os difeomorfismos estruturalmente estáveis formam um subconjunto aberto e denso de $D_{i f^{1}}\left(S^{1}\right)$. 


\section{Capítulo 2}

\section{Principais resultados}

Sejam $X \in \mathcal{H}\left(\mathbb{R}^{n}\right)$ com índice $n$ (ou 0 ) e $U$ um aberto de $\mathbb{R}^{n}$ contendo a origem. Denotemos por $\operatorname{Difr}^{r}(U, X), r \geq 1$, o conjunto dos $C^{r}$-difeomorfismos locais $f: U \rightarrow \mathbb{R}^{n}$ que preservam órbitas de $X$. Note que $0 \in f(U)$.

Sejam $U_{0}=U-\{0\}$ e $\frac{U_{0}}{X}$ o espaço das órbitas de $X$ com a topologia quociente obtido ao identificar cada órbita de $\mathrm{X}$ a um ponto. $\mathrm{O}$ espaço $\frac{U_{0}}{X}$ admite uma estrutura natural de variedade diferenciável $C^{\infty}$ de dimensão $n-1$, não necessariamente Hausdorff. Da Observação 1.1 do Capítulo 1 segue que $\frac{U_{0}}{X}$ pode ser realizada como sendo a $S^{n-1}$. Cada $f \in \operatorname{Dif}^{r}(U, X)$ induz um $C^{r}$-difeomorfismo local, $\bar{f}$ : $\frac{U_{0}}{X} \rightarrow \frac{\mathbb{R}_{0}^{n}}{X}$ de tal maneira que o seguinte diagrama comuta:

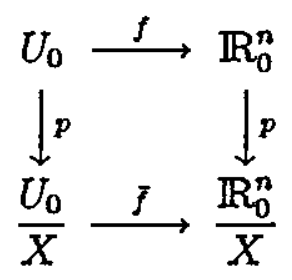

onde $p$ é a aplicação quociente.

Neste capítulo iremos enunciar e provar os dois principais teoremas encontrados 
em [1] (enunciados aqui como Teorema 2.1 e Teorema 2.2) e os lemas necessários para tanto. Tais teoremas nos dão informações a respeito de $\bar{f}$, fazendo um estudo da mesma via cartas. Primeiramente veremos dois lemas e também a prova do primeiro teorema para o caso particular $n=2$, a qual nos ajudará a entender a prova geral.

\subsection{Considerações iniciais e lemas preliminares}

Observaçāo 2.1 Seja $X \in \mathcal{H}\left(\mathbb{R}^{n}\right), n \geq 2$, com índice $n$ (ou 0 ) e cuja matriz é diagonalizável. Denotando por $\left\{\mu_{1}, \mu_{2}, \ldots, \mu_{k}\right\}, k \leq n$, o conjunto dos autovalores do campo $X$ onde $\mu_{j}=\alpha_{j} \pm i \beta_{j}, \operatorname{com} \alpha_{j}, \beta_{j} \in \mathbb{R}$, supomos que $\left|\alpha_{1}\right|<\left|\alpha_{j}\right|, \quad \forall j=$ $2, \ldots, k$. Sendo $E_{j}$ o subespaço invariante por $X$ associado a $\mu_{j}, j=1,2, \ldots, k$, supomos ainda que $\operatorname{dim} E_{j}=1$ ou 2 , conforme $\beta_{j}=0$ ou $\beta_{j} \neq 0$. Seja $E$ o subespaço complementar de $E_{1}$, isto é,

$$
E=\bigoplus_{j \neq 1} E_{j}
$$

Note que $\mathbb{R}^{n}=E_{1} \oplus E_{2} \oplus \cdots \oplus E_{k}$.

Exemplo 2.1 Seja $X \in \mathcal{H}\left(\mathbb{R}^{2}\right)$ com autovalores $\alpha_{1}$ e $\alpha_{2}$ tais que $\alpha_{2}<\alpha_{1}<0$. Então $E=E_{2}$, conforme Figura 2.1 .

Observaçāo 2.2 Seja $\left\{v_{1}, \ldots, v_{k}\right\}$ base para $\mathbb{R}^{n}$, onde $\left\{v_{j}\right\}$ é base para $E_{j}$ tal que:

- se $\operatorname{dim} E_{j}=1, v_{j}$ é um autovetor associado a $\mu_{j}$;

- se $\operatorname{dim} E_{j}=2, v_{j}=\left(v_{j 1}, v_{j 2}\right)$ onde $v_{j 1}+i v_{j 2}$ é um autovetor associado a $\alpha_{j}+i \beta_{j}$.

Dado $x \in \mathbb{R}^{n}$, sejam $\left(x_{1}, \ldots, x_{k}\right)$ as coordenadas de $x$ com relação à base $\left\{v_{1}, \ldots, v_{k}\right\}$, sendo $x_{j}=\left(x_{j 1}, x_{j 2}\right)$ se $\operatorname{dim} E_{j}=2$. 


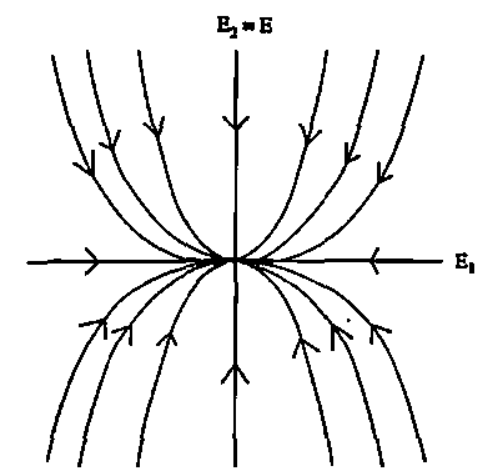

Figura 2.1: $O$ campo $X$ do Exemplo 2.1. Neste caso $E=E_{2}$, onde $E_{2}$ é o subespaço invariante por $X$ associado a $\alpha_{2}$.

Definimos o seguinte subconjunto do $R^{n}$ :

\section{Definição 2.1}

$$
\sum=\left\{\left(x_{1}, \ldots, x_{k}\right) \in E_{1} \times \ldots \times E_{k} ;\left|x_{1}\right|=1\right\} .
$$

Sendo $E$ como na Observação 2.1, obtemos

$$
\sum= \begin{cases}\{-1,1\} \times E & \text { se } \beta_{1}=0 \\ S^{1} \times E & \text { se } \beta_{1} \neq 0 .\end{cases}
$$

Seja $\pi: \mathbb{R}^{n}-E \longrightarrow \Sigma$ a projeção ao longo das órbitas de $X$. Dos resultados sobre campos lineares vistos no Capítulo 1 temos que $\pi$ está bem definida e é sobre. Veremos no Lema 2.2 que $E$ é invariante por $f$. Logo, definimos a aplicação

$$
\tilde{f}: \Sigma \rightarrow \Sigma
$$

de tal maneira que o seguinte diagrama comuta:

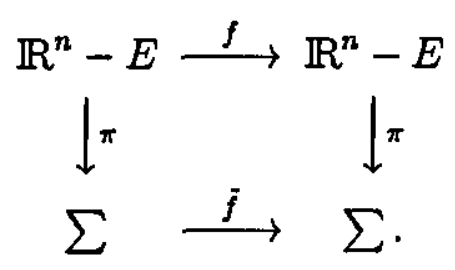


A próxima figura exemplifica a situação descrita acima para o caso em que $n=2$.



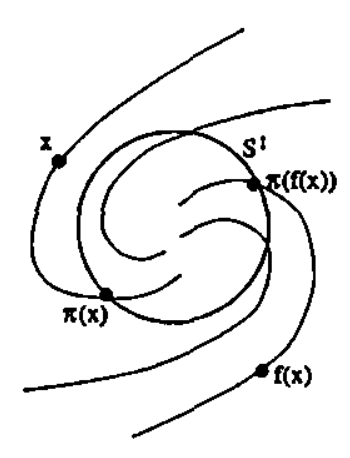

(ii)

Figura 2.2: As órbitas do Campo $X$ e o espaço $\Sigma$ para o caso em que (i) os autovalores são reais distintos e de mesmo sinal ou (ii) os autovalores são complexos conjugados. Como o diagrama anterior é comutativo, segue que $\pi(f(x))=\tilde{f}(\pi(x))$.

Estudaremos a aplicação $\tilde{f}: \Sigma \rightarrow \Sigma$ visto que a partir desta poderemos tirar conclusões a respeito de $\vec{f}: \frac{U_{0}}{X} \rightarrow \frac{\mathbb{R}_{0}^{n}}{X}$, desde que $\bar{f}=\pi \tilde{f} \pi^{-1}$, onde $\pi$ é a projeção ao longo das órbitas de $X$. Observe que para o caso em que $n=2, \frac{U_{0}}{X}$ pode ser realizada como a $S^{1}$.

Temos então os dois principais resultados enunciados abaixo.

Teorema 2.1 Seja $f \in \operatorname{Dif}^{f}(U, X), r \geq 1$, com $X \in \mathcal{H}\left(\mathbb{R}^{n}\right)$ como na Observação 2.1. Supondo que $\alpha_{k}<\ldots<\alpha_{1}<0$ então a aplicação $\tilde{f}: \Sigma \rightarrow \Sigma$ induzida de $f$ é dada como segue:

A. Se $\beta_{1}=0$, então $\tilde{f}=\left(\tilde{f}_{2}, \ldots, \tilde{f}_{k}\right)$ é dada por:

- se $\frac{\partial f_{1}}{\partial x_{1}}(0)>0:\left\{\begin{array}{l}\tilde{f}_{2}\left(x_{2}, \ldots, x_{k}\right)=M_{2} x_{2}+\varphi_{0}, \quad \varphi_{0}=c t e \\ \tilde{f}_{j}\left(x_{2}, \ldots, x_{k}\right)=M_{j} x_{j}+\varphi_{j}\left(x_{2}, \ldots, x_{j-1}\right) \quad 3 \leq j \leq k ;\end{array}\right.$

- se $\frac{\partial f_{1}}{\partial x_{1}}(0)<0:$ a mesma conclusão para $\tilde{f}^{2}$. 
B. Se $\beta_{1} \neq 0$, então $\tilde{f}=\left(\tilde{f}_{\theta}, \tilde{f}_{2}, \ldots, \tilde{f}_{k}\right)$ é dada por:

$$
\begin{aligned}
& \tilde{f}_{\theta}\left(\theta, x_{2}, \ldots, x_{k}\right)=\theta+\theta_{0} ; \\
& \tilde{f}_{j}\left(\theta, x_{2}, \ldots, x_{k}\right)=M_{j} x_{j}+\varphi_{j}\left(x_{2}, \ldots, x_{j-1}\right) 2 \leq j \leq k, \\
& \text { onde } \theta_{0} \in \mathbb{R} \text { e } M_{j}= \begin{cases}m_{j} \in \mathbb{R} & \text { se } \beta_{j}=0 \\
\left(\begin{array}{cc}
m_{j} & n_{j} \\
-n_{j} & m_{j}
\end{array}\right), m_{j}, n_{j} \in \mathbb{R} & \text { se } \beta_{j} \neq 0 .\end{cases}
\end{aligned}
$$

O próximo resultado diz que, adicionando algumas hipóteses ao Teorema 2.1, podemos concluir que $\tilde{f}$ é linear em todas as suas coordenadas se $\beta_{1}=0$ ou que $\tilde{f}$ é uma rotação na primeira coordenada e linear nas demais se $\beta_{1} \neq 0$.

Teorema 2.2 Suponha, além das hipóteses do Teorema 2.1, que os autovalores de $X$ satisfazem as condiçöes de não-ressonância e que $f \in C^{l}, l>\frac{\left|\alpha_{k}\right|}{\left|\alpha_{1}\right|}$. Então:

A. Se $\beta_{1}=0$ então $\tilde{f}: \Sigma \rightarrow \Sigma$ é linear.

B. Se $\beta_{1} \neq 0$ então $\tilde{f}=\left(\tilde{f}_{\theta}, \tilde{f}_{2}, \ldots, \tilde{f}_{k}\right)$, onde $\tilde{f}: S^{1} \times E \rightarrow S^{1} \times E$, é dada por:

$$
\begin{aligned}
& \tilde{f}_{\theta}\left(\theta, x_{2}, \ldots, x_{k}\right)=\theta+\theta_{0} ; \\
& \tilde{f}_{j}\left(\theta, x_{2}, \ldots, x_{k}\right) \text { é linear, } 2 \leq j \leq k,
\end{aligned}
$$

onde $\theta_{0} \in \mathbb{R}$.

O Teorema 3.5 de [2] é uma aplicação direta dos Teoremas 2.1 e 2.2, caracterizando os difeomorfismos $\bar{f}: \frac{U_{0}}{X} \rightarrow \frac{\mathbb{R}_{0}^{n}}{X}$ que são de Morse-Smale e, portanto, estruturalmente estáveis.

Para provarmos estes dois teoremas, precisamos primeiramente de dois lemas.

Lema 2.1 Sejam $X$ e $Y$ campos de vetores $C^{1}$ em $\mathbb{R}^{n}$ tais que $X(p)=Y(q)=0$. Suponhamos que $f:\left(\mathbb{R}^{n}, p\right) \rightarrow\left(\mathbb{R}^{n}, q\right)$ é um germe de um difeomorfismo $C^{1}$ que envia órbitas de $X$ em órbitas de $Y$. Sejam $A=D X_{p}, B=D Y_{q}$ e $J=D f_{p}$. Supondo ainda que $A$ e $B$ são não-singulares então: 
i) $A=k J^{-1} B J$, onde $k \in \mathbb{R}$;

ii) $S e X=Y$ e $p=q$ então $k= \pm 1$;

iii) $S e X=Y, p=q$ e existir um autovalor $\lambda$ de $A$ tal que $-\lambda$ não é autovalor de $A$, então $k=1$.

\section{Demonstraçāo:}

Observe que a condição de que $A$ e $B$ são não-singulares nos diz que $p$ e $q$ são singularidades isoladas de $X$ e $Y$, respectivamente (isto segue do Teorema da Função Inversa).

i) Vamos supor, sem perda de generalidade, que $p=q=0$. Como $f$ envia órbitas de $X$ em órbitas de $Y$, temos que

$$
D f_{x}(X(x))=k(x) Y(f(x))
$$

onde $k:\left(\mathbb{R}^{n}, 0\right) \rightarrow(\mathbb{R}, 0)$ é contínua fora de $0 \in \mathbb{R}^{n}$. Temos então que

$$
D f_{t x}\left(\frac{1}{t} X(t x)\right)=k(t x)\left(\frac{1}{t} Y(f(t x))\right) .
$$

Faremos $t \rightarrow 0$ nesta última expressão. Como

$$
\lim _{t \rightarrow 0} D f_{t x}=D f_{0}=J \quad \text { e } \quad \lim _{t \rightarrow 0} \frac{1}{t} X(t x)=\lim _{t \rightarrow 0} \frac{X(t x)-X(0)}{t}=D X_{0} \cdot x=A \cdot x
$$

temos que

$$
\lim _{t \rightarrow 0}\left[D f_{t x}\left(\frac{1}{t} X(t x)\right)\right]=J . A . x
$$

Além disso,

$$
\lim _{t \rightarrow 0} \frac{1}{t} Y(f(t x))=\lim _{t \rightarrow 0} \frac{Y(f(t x))-Y(f(0))}{t}=D(Y \circ f)_{0} . x=D Y_{f(0)} D f_{0} \cdot x=B . J . x .
$$

Portanto, como $A, B$ e $J$ são não singulares, concluímos que $\underset{t \rightarrow 0}{\exists} k(t x)$ e que

$$
J . A . x=\left(\lim _{t \rightarrow 0} k(t x)\right) B . J . x .
$$


Como $\lim _{t \rightarrow 0} k(t x)$ não depende de $t$, escrevemos $\lim _{t \rightarrow 0} k(t x)=\sigma(x)$. Assim,

$$
J A x=\sigma(x) B J x
$$

Resta então mostrarmos que $\sigma(x)$ é constante, $\forall x \in \mathbb{R}^{n}$.

Sejam $x, y \in \mathbb{R}^{n}$ linearmente independentes (l.i.). Então $B J x$ e $B J y$ também são l.i. e, usando 2.1 , temos:

$$
\begin{aligned}
\sigma(x) B J x+\sigma(y) B J y & =J A x+J A y=J A(x+y)=\sigma(x+y) B J(x+y)= \\
& =\sigma(x+y) B J x+\sigma(x+y) B J y .
\end{aligned}
$$

Assim,

$$
[\sigma(x)-\sigma(x+y)] B J x+[\sigma(y)-\sigma(x+y)] B J y=0,
$$

ou seja, $\sigma(x)=\sigma(y)$, como queríamos.

Observe que se $x$ e $y$ forem linearmènte dependentes, basta tomar $z \in \mathbb{R}^{n}$ tal que tanto $\{x, z\}$ quanto $\{y, z\}$ sejam li.i. e o resultado segue da transitividade de $\mathbb{R}$. Portanto $\sigma(x)$ é constante, $\forall x \in \mathbb{R}^{n}$, ou seja, $A=k J^{-1} B J$, com $k \in \mathbb{R}$.

ii) Supondo que $X=Y$ e $p=q=0$, mostremos que $k= \pm 1$. Sejam $\tilde{A}$ e $\tilde{J}$ as complexificações de $A$ e $J$, respectivamente. Vamos supor, por absurdo, que $|k|<1$.

Seja $\lambda$ o autovalor de $\tilde{A}$ de maior norma (isto é, $|\beta| \leq|\lambda|, \forall \beta$ autovalor de $\tilde{A}$ ) e seja $v$ um autovetor de $\tilde{A}$ associado a $\lambda$. Por (i) temos que $\tilde{J} \tilde{A}=k \tilde{A} \tilde{J}$. Assim,

$$
\tilde{A}(\tilde{J} v)=(\tilde{A} \tilde{J}) v=k^{-1} \tilde{J} \tilde{A} v=k^{-1} \tilde{J} \lambda v=\left(k^{-1} \lambda\right) \tilde{J} v
$$

ou seja, $k^{-1} \lambda$ é autovalor de $\tilde{A}$. Como $|k|<1$, temos que $\left|k^{-1} \lambda\right|>|\lambda|$. 
Absurdo pois $\lambda$ é o autovalor de $\tilde{A}$ de maior norma. Portanto $|k| \geq 1$. Supondo $|k|>1$, com um raciocínio análogo também chegaremos a um absurdo. Consequentemente $k= \pm 1$.

iii) Suponha que $X=Y$ e que $\lambda$ seja um autovalor de $\tilde{A}$ tal que $-\lambda$ não é autovalor de $\tilde{A}$. Sabemos por (ii) que $k= \pm 1$, ou seja, $\tilde{J} \tilde{A}= \pm \tilde{A} \tilde{J}$. Vamos supor que $k=-1$. Seja $v$ um autovetor de $\tilde{A}$ associado a $\lambda$. Temos,

$$
\tilde{A}(\tilde{J} v)=(\tilde{A} \tilde{J}) v=-(\tilde{J} \tilde{A}) v=-\tilde{J}(\tilde{A} v)=-\tilde{J}(\lambda v)=-\lambda(\tilde{J} v)
$$

ou seja, $-\lambda$ é autovalor de $\tilde{A}$. Absurdo: Logo $k=1$.

Observaçāo 2.3 Concluímos deste Lema que se $f \in \operatorname{Dif}^{r}(U, X)$, onde $X \in \mathcal{H}\left(\mathbb{R}^{n}\right)$ tem índice $n$ ou 0 , então as hipóteses do item (iii) estão satisfeitas e daí $X\left(D f_{0}\right)=\left(D f_{0}\right) X$.

Lema 2.2 Seja $f \in D i f^{r}(U, X)$, onde $X \in \mathcal{H}\left(\mathbb{R}^{n}\right)$ é um campo de vetores como na Observação 2.1 e $U$ é uma vizinhança da origem em $\mathbb{R}^{n}$. Entäo E é invariante por $f$, ou seja, $f(U \cap E) \subset E$.

\section{Demonstraçāo:}

Pela Observação 2.3 temos que $\left(D f_{0}\right) X=X\left(D f_{0}\right)$. Assim, como $E$ é invariante por $\mathrm{X}$, segue que $E$ também é invariante por $D f_{0}$, ou seja, $D f_{0} . E=E$. Temos então que $f(U \cap E)$ é tangente a $E$ em $0 \in \mathbb{R}^{n}$.

Dado $x \notin E$, seja $\rho_{x}(t) \circ$ ângulo entre $X\left(X_{t}(x)\right), t \in \mathbb{R}$ e $E_{1}$. Como 0 é um poço e $\left|\alpha_{1}\right|<\left|\alpha_{j}\right|, 2 \leq j \leq k$, temos que $X_{t}(x) \rightarrow 0$ quando $t \rightarrow \infty$, de tal maneira que as trajetórias do campo "tangenciam $E_{1}$ em 0" (de fato, do Teorema 1.2 do Cap.1 temos 
que $X_{t}(x)=e^{t X} x$. Como $\left|\alpha_{1}\right|<\left|\alpha_{j}\right|$ e $x \notin E$, segue que a primeira coordenada de $X_{t}(x)$ tende a zero mais lentamente do que as demais coordenadas). Portanto $\lim _{t \rightarrow \infty} \rho_{x}(t)=0$.

Seja $x \in f(U \cap E)$. Se $f(U \cap E) \not \subset E$ então $x \notin E$ e como vimos acima, $\lim _{t \rightarrow \infty} \rho_{x}(t)=0$. Mas $f(U \cap E)$ é tangente a $E$ em $0 \in \mathbb{R}^{n}$ e $\mathcal{O}_{X}(x) \subset E \Rightarrow$ $f\left(\mathcal{O}_{X}(x)\right) \subset f(E)$, o que nos diz que $\lim _{t \rightarrow \infty} \rho_{x}(t) \neq 0$. Contradição! Portanto $x \in E$, como queríamos.

Veja novamente o exemplo 2.1. O Lema 2.2 nos diz que $f$ leva $E_{2}$ sobre $E_{2}$.

\subsection{Prova do Teorema 2.1}

Como a demonstração do Teorema 2.1 é bastante trabalhosa, estudaremos primeiro o caso $n=2$ para $X$ e $f$ nas condições do Teorema 2.1.

Proposiçāo 2.1 Sejam $f \in D i f^{r}(U, X), r \geq 1, U$ aberto de $\mathbb{R}^{2}$ contendo a origem e $X$ um campo de vetores linear hiperbólico em $\mathbb{R}^{2}$.

$A$ - Se os autovalores do campo $X$ são reais distintos e de mesmo sinal então ou $\tilde{f}: \Sigma \rightarrow \Sigma$ ou $\tilde{f}^{2}: \Sigma \rightarrow \Sigma$ é afim;

$B$ - Se os autovalores de $X$ são complexos conjugados então $\tilde{f}: S^{1} \rightarrow S^{1}$ é uma rotação.

\section{Demonstraçāo:}

CASO A - Sejam $\alpha_{1}$ e $\alpha_{2}$ os autovalores de $X$ tais que $\alpha_{2}<\alpha_{1}<0$. Neste caso $X=\left(\begin{array}{cc}\alpha_{1} & 0 \\ 0 & \alpha_{2}\end{array}\right)$ e suas órbitas são dadas pela Figura 2.1.

Escrevemos $f(x)=\left(f_{1}(x), f_{2}(x)\right)$, onde $f_{i}: U \subset \mathbb{R}^{2} \longrightarrow E_{i}, \quad i=1,2 \mathrm{e}$ $x=\left(x_{1}, x_{2}\right)$. Como $f$ é diferenciável e está definida em uma vizinhança da origem 
temos:

$$
f(x)=f^{\prime}(0,0) \cdot x+R(x)
$$

com

$$
\lim _{x \rightarrow 0} \frac{R(x)}{\|x\|}=0 .
$$

$$
\begin{aligned}
& \text { Pela Observação } 2.3 \text { temos que } f^{\prime}(0,0) \text { comuta com } X \text {. Logo, temos que } f^{\prime}(0,0)= \\
& \left(\begin{array}{ll}
a & 0 \\
0 & b
\end{array}\right) \text { onde } a=\frac{\partial f_{1}}{\partial x_{1}}(0,0) \text { e } b=\frac{\partial f_{2}}{\partial x_{2}}(0,0) \text {. Portanto } \\
& \qquad f_{1}\left(x_{1}, x_{2}\right)=a x_{1}+R_{1}\left(x_{1}, x_{2}\right) \text { e } f_{2}\left(x_{1}, x_{2}\right)=b x_{2}+R_{2}\left(x_{1}, x_{2}\right)
\end{aligned}
$$

onde

$$
\lim _{x \rightarrow 0} \frac{R_{i}(x)}{\|x\|}=0, \quad i=1,2
$$

Como $\Sigma=\{ \pm 1\} \times E_{2}$, seja $\Sigma_{1}=\{1\} \times E_{2}$ e $\Sigma_{-1}=\{-1\} \times E_{2}$.

Sejam $H_{+}=\left\{\left(x_{1}, x_{2}\right) \in E_{1} \times E_{2} ; x_{1}>0\right\}$ e $H_{-}=\left\{\left(x_{1}, x_{2}\right) \in E_{1} \times E_{2} ; x_{1}<0\right\}$. Se $a>0 \quad(a<0)$ então $\tilde{f}\left(\Sigma_{1}\right) \subset \Sigma_{1}$ e $\tilde{f}\left(\Sigma_{-1}\right) \subset \Sigma_{-1} \quad\left(\tilde{f}^{2}\left(\Sigma_{1}\right) \subset \Sigma_{1}\right.$ e $\tilde{f}^{2}\left(\Sigma_{-1}\right) \subset$ $\left.\Sigma_{-1}\right)$. De fato, para concluir que $\tilde{f}\left(\Sigma_{1}\right) \subset \Sigma_{1}$ basta mostrarmos que $f\left(H_{+}\right) \subset H_{+}$. Seja $\left(x_{1}, x_{2}\right) \in H_{+}$. Então, como $X_{t}\left(x_{1}, x_{2}\right) \subset H_{+}$, é suficiente analisarmos para $\left(x_{1}, x_{2}\right)$ muito próximo da origem. Nestas condições temos que $a x_{1}>\left|R_{1}\left(x_{1}, x_{2}\right)\right|$, visto que $R_{1}\left(x_{1}, x_{2}\right)$ tem termos de ordem maior do que 1 e $x_{1}, x_{2}<1$. Assim, $f_{1}\left(x_{1}, x_{2}\right)=a x_{1}+R_{1}\left(x_{1}, x_{2}\right)>0$, de onde concluímos que $f\left(x_{1}, x_{2}\right) \in H_{+}$. Analogamente, mostra-se que $f\left(H_{-}\right) \subset H_{-}$. Se $a<0$, o mesmo se mostra para $\tilde{f}^{2}$.

Portanto, se $a>0$ então $\Sigma_{1}$ e $\Sigma_{-1}$ são invariantes por $\tilde{f}$ e, se $a<0$ então o mesmo acontece com relação a $\tilde{f}^{2}$. Iremos estudar os dois casos: $a>0$ e $a<0$. Veremos que, se $a>0$ então $\tilde{f}$ é afim e, se $a<0$, então $\tilde{f}^{2}$ é afim. 
Vamos supor primeiramente que $a>0$.

Seja $(1, \tau) \in \Sigma_{1}$. Denotaremos às vezes $(1, \tau) \in \Sigma_{1}$ simplesmente por $\tau$. A órbita de $X$ por $(1, \tau) \in \Sigma_{1}$ é dada por

$$
X_{t}(\tau)=\left(e^{\alpha_{1} t}, e^{\alpha_{2} t} \tau .\right)
$$

Escreveremos a órbita de $X$ em função de um outro parâmetro. Façamos $u=$ $e^{\alpha_{1} t}$. Então $t=\frac{1}{\alpha_{1}} \ln u$ e assim $e^{\alpha_{2} t} \tau=u^{\frac{\alpha_{2}}{\alpha_{1}}} \tau$.

Obtemos então uma nova expressão para a órbita de $X$, na qual o parâmetro é a primeira coordenada:

$$
X_{u}(\tau)=\left(u, u^{\left.\frac{\alpha_{2}}{\alpha_{1}} \tau\right)}\right.
$$

Observe que como $\alpha_{2}<\alpha_{1}<0$ temos que $\frac{\alpha_{2}}{\alpha_{1}}>1$. De 2.3 obtemos:

$$
f\left(X_{u}(\tau)\right)=f\left(u, u^{\frac{\alpha_{2}}{\alpha_{1}} \tau}\right)=\left(a u+R_{1}\left(u, u^{\frac{\alpha_{2}}{\alpha_{1}}} \tau\right), b u^{\frac{\alpha_{2}}{\alpha_{1}}} \tau+R_{2}\left(u, u^{\frac{\alpha_{2}}{\alpha_{1}}} \tau\right)\right) .
$$

Como $f$ transforma a órbita de $X$ pelo ponto $(1, \tau)$ na órbita pelo ponto $\tilde{f}(\tau)$, segue de 2.4 e de 2.5 que:

$$
f\left(X_{u}(\tau)\right)=\left(a u+R_{1}\left(u, u^{\frac{\alpha_{2}}{\alpha_{1}}} \tau\right),\left[a u+R_{1}\left(u, u^{\left.\left.\frac{\alpha_{2}}{\alpha_{1}} \tau\right)\right]^{\frac{\alpha_{2}}{\alpha_{1}}}} \tilde{f}(\tau)\right) .\right.\right.
$$

Comparando 2.5 e 2.6 temos:

$$
b u^{\frac{\alpha_{2}}{\alpha_{1}}} \tau+R_{2}\left(u, u^{\frac{\alpha_{2}}{\alpha_{1}}} \tau\right)=\left[a u+R_{1}\left(u, u^{\left.\frac{\alpha_{2}}{\alpha_{1}} \tau\right)}\right]^{\frac{\alpha_{2}}{\alpha_{1}}} \tilde{f}(\tau) .\right.
$$

Multiplicando ambos os membros por $u^{-\frac{\alpha_{2}}{\alpha_{1}}}$ obtemos:

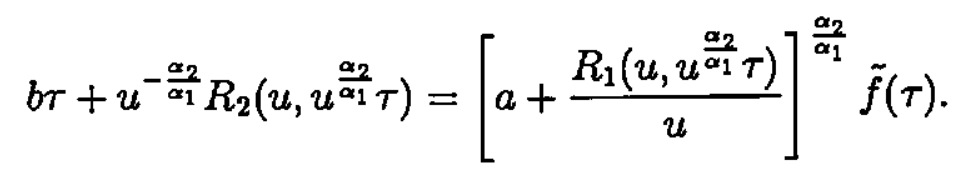

Derivando com relação a $\tau$ :

$$
\begin{aligned}
b+D_{2} R_{2}\left(u, u^{\frac{\alpha_{2}}{\alpha_{1}}} \tau\right)=\frac{\alpha_{2}}{\alpha_{1}}\left[a u+R_{1}\left(u, u^{\left.\left.\frac{\alpha_{2}}{\alpha_{1}} \tau\right)\right]^{\frac{\alpha_{2}}{\alpha_{1}}-1}} D_{2} R_{1}\left(u, u^{\left.\frac{\alpha_{2}}{\alpha_{1}} \tau\right) \tilde{f}(\tau)+}\right.\right.\right. \\
+\left[a+\frac{R_{1}\left(u, u^{\left.\frac{\alpha_{2}}{\alpha_{1}} \tau\right)}\right.}{u}\right]^{\frac{\alpha_{2}}{\alpha_{1}}} \tilde{f}^{\prime}(\tau) .
\end{aligned}
$$


O passo seguinte será calcular o limite da expressão 2.7 quando $u \rightarrow 0$. De 2.3 segue que $D_{2} f_{2}\left(x_{1}, x_{2}\right)=b+D_{2} R_{2}\left(x_{1}, x_{2}\right)$. Como $f \in C^{r}, r \geq 1$, temos também que $\lim _{\left(x_{1}, x_{2}\right) \rightarrow(0,0)} D_{2} f_{2}\left(x_{1}, x_{2}\right)=D_{2} f_{2}(0,0)=b$.

Portanto,

$$
\lim _{u \rightarrow 0} D_{2} R_{2}\left(u, u^{\frac{\alpha_{2}}{\alpha_{1}}} \tau\right)=0
$$

Analogamente,

$$
\lim _{u \rightarrow 0} D_{2} R_{1}\left(u, u^{\frac{\alpha_{2}}{\alpha_{1}}} \tau\right)=0
$$

pois, $D_{2} f_{1}\left(x_{1}, x_{2}\right)=D_{2} R_{1}\left(x_{1}, x_{2}\right)$ e $\lim _{\left(x_{1}, x_{2}\right) \rightarrow(0,0)} D_{2} f_{1}\left(x_{1}, x_{2}\right)=D_{2} f_{1}(0,0)=0$.

Temos ainda que

$$
\lim _{u \rightarrow 0} \frac{R_{1}\left(u, u^{\left.\frac{\alpha_{2}}{\alpha_{1}} \tau\right)}\right.}{u}=0
$$

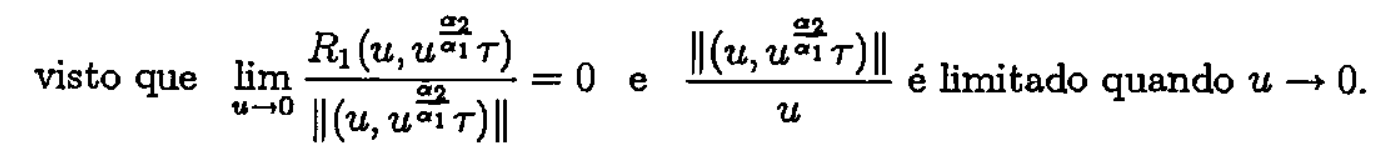

Assim, fazendo $u \rightarrow 0$ em 2.7 e usando $2.8,2.9$ e 2.10 , obtemos $b=a^{\frac{\alpha_{2}}{\alpha_{1}}} \tilde{f}^{\prime}(\tau)$ e portanto,

$$
\tilde{f}(\tau)=a^{-\frac{\alpha_{2}}{\alpha_{1}} b r+c}
$$

ou seja, $\tilde{f}$ é afim.

Note que $\frac{\alpha_{2}}{\alpha_{1}}-1>0$, o que foi fundamental para esta conclusão.

\section{$\underline{\text { Vamos supor agora que } a<0}$.}

Então $f^{2}\left(H_{+}\right) \subset H_{+}$e $f^{2}\left(H_{-}\right) \subset H_{-}$e assim quando $a<0, f^{2}$ está nas mesmas condições que $f$ quando $a>0$. Deste modo, com raciocínio análogo ao anterior, temos que $\tilde{f}^{2}(\tau)=A^{-\frac{\alpha_{2}}{\alpha_{1}}} B \tau+C$. Fą̧amos $f^{2}=g$. Então $A=\frac{\partial g_{1}}{\partial x_{1}}(0,0)$, 
$B=\frac{\partial g_{2}}{\partial x_{2}}(0,0)$ e $C$ é constante. Temos que $g: \mathbb{R}^{2} \longrightarrow \mathbb{R}^{2}$ é tal que $g=\left(g_{1}, g_{2}\right)$, onde $g_{j}=f_{j} \circ f, \quad j=1,2$. Logo,

$$
A=\left[\frac{\partial f_{1}}{\partial x_{1}}(0,0)\right]^{2}+\frac{\partial f_{1}}{\partial x_{2}}(0,0) \frac{\partial f_{2}}{\partial x_{1}}(0,0)=a^{2}
$$

e

$$
B=\left[\frac{\partial f_{2}}{\partial x_{2}}(0,0)\right]^{2}+\frac{\partial f_{2}}{\partial x_{1}}(0,0) \frac{\partial f_{1}}{\partial x_{2}}(0,0)=b^{2},
$$

de onde concluímos que

$$
\tilde{f}^{2}(\tau)=\left(a^{-\frac{\alpha_{2}}{\alpha_{1}}}\right)^{2} b^{2} \tau+c
$$

ou seja, $\bar{f}^{2}$ é afim.

CASO B - Suponhamos que $X$ possua autovalores complexos conjugados $\mu=$ $\alpha \pm i \beta, \alpha \neq 0$. Então as órbitas do campo $X=\left(\begin{array}{rr}\alpha & \beta \\ -\beta & \alpha\end{array}\right)$ são dadas pela Figura 2.3 .

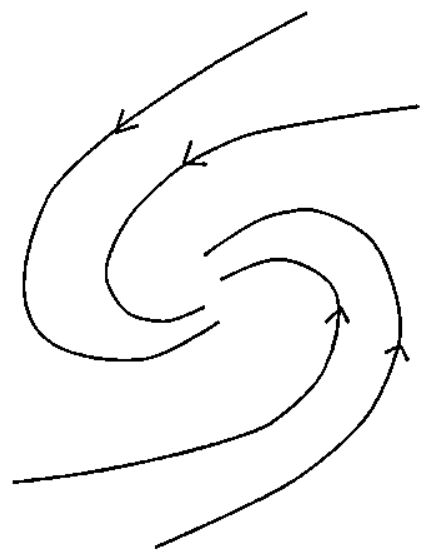

Figura 2.3: As órbitas do campo $X \in \mathfrak{X}\left(\mathbb{R}^{2}\right)$ quando seus autovalores sāo complexos conjugados.

De modo análogo ao anterior, pondo $x=\left(x_{1}, x_{2}\right)$, temos:

$$
f(x)=f^{\prime}(0) \cdot x+R(x) \quad \text { com } \quad \lim _{x \rightarrow 0} \frac{R(x)}{\|x\|}=0 .
$$


Como $f^{\prime}(0)$ comuta com $X$ temos que $f^{\prime}(0)=A=\left(\begin{array}{rr}a & b \\ -b & a\end{array}\right)$.

Portanto,

$$
f(x)=A x+R(x)
$$

Como $\Sigma=S^{1}$, temos $\tilde{f}: S^{1} \longrightarrow S^{1}$. Denotaremos $\tau \in S^{1}$ por $(\cos \theta, \operatorname{sen} \theta)$ e $\tilde{f}(\tau) \in S^{1}$ por $\tilde{f}_{\theta}(\tau)$.

$$
\begin{gathered}
\text { Sendo } B(t)=\left(\begin{array}{rr}
\cos t & \operatorname{sen} t \\
-\operatorname{sen} t & \cos t
\end{array}\right), \text { a trajetória de } X \text { por } \tau \text { é dada por } \\
X_{t}(\tau)=e^{\alpha t} B(\beta t) \tau .
\end{gathered}
$$

De maneira análoga a que fizemos no caso $\mathrm{A}$, escreveremos a órbita de $X$ em função de um novo parâmetro, $r=\left\|X_{t}(\tau)\right\|$. Então $r=e^{\alpha t}$, ou seja, $t=\frac{1}{\alpha} \ln r$. Substituindo em 2.12 obtemos:

$$
e^{\alpha t} B(\beta t) \tau=r B(\bar{\beta} \ln r) \tau, \quad \text { onde } \bar{\beta}=\frac{\beta}{\alpha} .
$$

Obtemos portanto uma nova expressão para a órbita do campo $X$, cujo parâmetro é $r$ :

$$
X_{r}(\tau)=r B(\ddot{\beta} \ln r) \tau
$$

De 2.11 segue que:

$$
f\left(X_{r}(\tau)\right)=A r B(\bar{\beta} \ln r) \tau+R\left(X_{r}(\tau)\right)
$$

Como $f$ transforma a órbita de $X$ pelo ponto $\tau$ na órbita pelo ponto $\tilde{f}_{\theta}(\tau)$, segue de 2.13 que:

$$
f\left(X_{r}(\tau)\right)=\left\|f\left(X_{r}(\tau)\right)\right\| B\left[\bar{\beta} \ln \left(\left\|f\left(X_{r}(\tau)\right)\right\|\right)\right] \tilde{f}_{\theta}(\tau)
$$


Encontremos $\|f(x)\|$. Seja $\rho(x)=\|f(x)\|$. Assim, $\rho(x)=\|A x+R(x)\|=\|A x\|+$ $\|A x+R(x)\|-\|A x\|$. Seja $\bar{R}(x)=\|A x+R(x)\|-\|A x\|$. Então $\rho(x)=\|A x\|+\bar{R}(x)$. Logo,

$$
\rho\left(X_{r}(\tau)\right)=\left\|A X_{r}(\tau)\right\|+\bar{R}\left(X_{r}(\tau)\right)=\delta r+\bar{R}\left(X_{r}(\tau)\right),
$$

onde $\delta=\sqrt{a^{2}+b^{2}}$. É fácil ver que $\lim _{x \rightarrow 0} \frac{\bar{R}(x)}{\|x\|}=0$.

Substituindo $\rho\left(X_{r}(\tau)\right)$ em 2.15 , obtemos:

$$
f\left(X_{r}(\tau)\right)=\left[\delta r+\bar{R}\left(X_{r}(\tau)\right)\right] B\left[\bar{\beta} \ln \left(\delta r+\bar{R}\left(X_{r}(\tau)\right)\right] \tilde{f}_{\theta}(\tau) .\right.
$$

Comparando 2.14 e 2.16 temos:

$$
A r B(\bar{\beta} \ln r) \tau+R\left(X_{r}(\tau)\right)=\left[\delta r+\bar{R}\left(X_{r}(\tau)\right)\right] B\left[\bar{\beta} \ln \left(\delta r+\bar{R}\left(X_{r}(\tau)\right)\right] \tilde{f}_{\theta}(\tau) .\right.
$$

Multiplicando esta última expressão por $r^{-1} B(-\bar{\beta} \ln r)$ obtemos:

$$
A \tau+B(-\bar{\beta} \ln r) \frac{R\left(X_{r}(\tau)\right)}{r}=\left[\delta+\frac{\bar{R}\left(X_{r}(\tau)\right)}{r}\right] B\left[\bar{\beta} \ln \left(\delta+\frac{\bar{R}\left(X_{r}(\tau)\right)}{r}\right)\right] \bar{f}_{\theta}(\tau) .
$$

Fazendo $r \rightarrow 0$ e observando que $B(-\bar{\beta} \ln r)$ é limitado, segue que:

$$
A \tau=\delta B(\bar{\beta} \ln \delta) \tilde{f}_{\theta}(\tau)
$$

Portanto,

$$
\tilde{f}_{\theta}(\tau)=\delta^{-1} B(-\widetilde{\beta} \ln \delta) A \tau .
$$

É fácil ver que $\delta^{-1} B(-\bar{\beta} \ln \delta) A$ é uma rotação. Assim, escrevemos:

$$
\tilde{f}_{\theta}\left(\theta_{0}\right)=\theta_{0}+\varphi_{0}
$$

onde $\varphi_{0}$ é constante. 
Observaçāo 2.4 Da Proposição 2.1 concluímos que:

- Se $\alpha_{2}<\alpha_{1}<0$ então ou $\tilde{f}$ não tem nenhum ponto periódico (caso em que $a^{-\frac{\alpha_{2}}{\alpha_{1}}} b=1$ e $\left.c \neq 0\right)$, ou todos os pontos de $\Sigma$ são periódicos de $\tilde{f}\left(a^{-\frac{\alpha_{2}}{\alpha_{1}}} b=1\right.$ e $c=0$ ) ou $\tilde{f}$ tem apenas dois pontos periódicos $\left(a^{-\frac{\alpha_{2}}{\alpha_{1}}} b \neq 1\right)$. Caso existam, os pontos periódicos têm período 1 ou 2 , conforme $a>0$ ou $a<0$, respectivamente.

- Se os autovalores de $X$ são complexos conjugados então ou todos os pontos de $S^{1}$ são pontos periódicos da rotação $\tilde{f}$, o que acontece se $\varphi_{0}$ é da forma $\frac{p \pi}{q}$ para $p, q \in \mathbb{Z}$, ou, se $\nexists p, q \in \mathbb{Z}$ tal que $\varphi_{0}=\frac{p \pi}{q}$ então a órbita de cada ponto é densa em $S^{1}$ e portanto, $\tilde{f}$ não tem pontos periódicos.

No Capítulo 3 faremos um estudo mais rigoroso de $\tilde{f}$ tirando a partir deste conclusões a respeito de $\bar{f}: \frac{U_{0}}{X} \rightarrow \frac{\mathbb{R}_{0}^{n}}{X}$.

Passemos agora à demonstração do Teorema 2.1.

\section{Demonstraçāo:}

CASO A: Seja $\beta_{1}=0$. Suponhamos que $\frac{\partial f_{1}}{\partial x_{1}}(0)>0$.

Consideremos o seguinte conjunto de autovalores do campo $X$ :

$$
\left\{\begin{array}{l}
\mu_{1}=\alpha_{1} \\
\mu_{2}=\alpha_{2} \pm i \beta_{2} \\
\vdots \\
\mu_{k}=\alpha_{k} \pm i \beta_{k}
\end{array}\right.
$$

$k \leq n$, com $\alpha_{k}<\ldots<\alpha_{1}<0$, onde o autoespaço $E_{1}$ associado a $\mu_{1}$ tem dimensão 1. Temos também que $\Sigma=\{ \pm 1\} \times E$, sendo $E=E_{2} \times \cdots E_{k}$. Lembre que $\mathbb{R}^{n}=E_{1} \oplus \cdots \oplus E_{k}$. 
Escrevemos $f: U \subset \mathbb{R}^{n} \rightarrow \mathbb{R}^{n}$ como $f=\left(f_{1}, \ldots, f_{k}\right)$, onde $f_{j}: U \subset \mathbb{R}^{n} \rightarrow E_{j}, \quad j=1, \ldots, k$. Como $f \in C^{r}, r \geq 1$, temos:

$$
f(x)=D f(0) \cdot x+R(x),
$$

onde $x=\left(x_{1}, \ldots, x_{k}\right)$ e $\lim _{x \rightarrow 0} \frac{R(x)}{\|x\|}=0$.

Como $D f(0)$ comuta com $X$ e $E_{j}$ é invariante por $X$, sendo

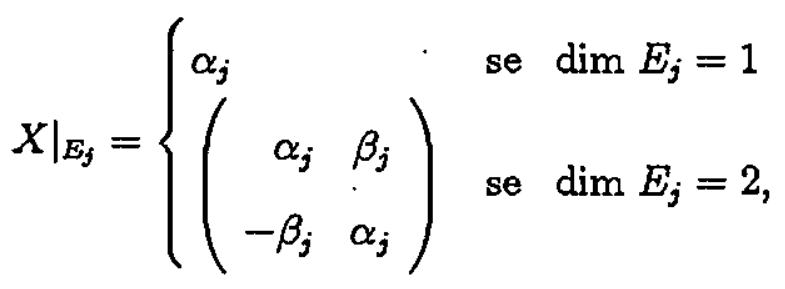

concluímos que $\left.D f(0)\right|_{E_{j}}=A_{j}= \begin{cases}a_{j} & \text { se } \operatorname{dim} E_{j}=1, \\ \left(\begin{array}{rr}a_{j} & b_{j} \\ -b_{j} & a_{j}\end{array}\right) & \text { se } \operatorname{dim} E_{j}=2 .\end{cases}$

Assim, temos que $f_{j}: U \subset \mathbb{R}^{n} \rightarrow E_{j}, \quad j=1, \ldots, k$ é dada por:

$$
f_{j}\left(x_{1}, \ldots, x_{k}\right)=A_{j} x_{j}+R_{j}\left(x_{1}, \ldots, x_{k}\right),
$$

onde $A_{j}$ é como acima e $\lim _{x \rightarrow 0} \frac{R_{j}(x)}{\|x\|}=0 \quad j=1, \ldots, k$.

Consideremos os seguintes subconjuntos do $\mathbb{R}^{n}$ :

$$
\begin{aligned}
H_{+} & =\left\{\left(x_{1}, \ldots, x_{k}\right) \in E_{1} \times \ldots \times E_{k} ; x_{1}>0\right\} \\
\Sigma_{1} & =\left\{\left(\tau_{1}, \tau_{2}, \ldots, \tau_{k}\right) \in \Sigma ; \quad \tau_{1}=1\right\} \\
\Sigma_{-1} & =\left\{\left(\tau_{1}, \tau_{2}, \ldots, \tau_{k}\right) \in \Sigma ; \quad \tau_{1}=-1\right\}
\end{aligned}
$$

Como $a_{1}=\frac{\partial f_{1}}{\partial x_{1}}(0)>0$ então $\tilde{f}\left(\Sigma_{1}\right) \subset \Sigma_{1}$ e $\tilde{f}\left(\Sigma_{-1}\right) \subset \Sigma_{-1}$. De fato, basta observar que $f\left(H_{+}\right) \subset H_{+}$(raciocínio análogo ao caso $n=2$ da proposição anterior). 
Denotemos $\tau \in \Sigma_{1}$ por $\left(\tau_{2}, \ldots, \tau_{k}\right)$ e escrevemos $\tilde{f}=\left(\tilde{f}_{2}, \ldots, \tilde{f}_{k}\right)$. A órbita de $X$ pelo ponto $\tau \in \Sigma_{1}$ é dada por:

$$
X_{t}(\tau)=\left(X_{t}^{1}(\tau), X_{t}^{2}(\tau), \cdots, X_{t}^{j}(\tau), \cdots, X_{t}^{k}(\tau)\right)
$$

onde $X_{t}^{1}(\tau)=e^{\alpha_{1} t} \quad$ e $\quad X_{t}^{j}(\tau)=\left\{\begin{array}{ll}e^{\alpha_{j} t} \tau_{j} & \text { se } \mu_{j} \in \mathbb{R} \\ e^{\alpha_{j} t} B\left(\beta_{j} t\right) \tau_{j} & \text { se } \mu_{j} \notin \mathbb{R}\end{array} \quad j=2, \ldots, k\right.$ com $B(t)=\left(\begin{array}{rr}\cos t & \operatorname{sen} t \\ -\operatorname{sen} t & \cos t\end{array}\right)$

Iremos escrever a órbita de $X$ usando como parâmetro a primeira coordenada $u=e^{\alpha_{1} t}$. Assim $t=\frac{1}{\alpha_{1}} \ln u$ e substituindo em 2.18 obtemos uma nova expressão para a órbita de $X$ :

$$
X_{u}(\tau)=\left(X_{u}^{1}(\tau), X_{u}^{2}(\tau), \cdots, X_{u}^{j}(\tau), \cdots, X_{u}^{k}(\tau)\right)
$$

onde $X_{u}^{1}(\tau)=u \quad$ e $\quad X_{u}^{j}(\tau)=\left\{\begin{array}{ll}u^{\bar{\alpha}_{j}} \tau_{j} & \text { se } \mu_{j} \in \mathbb{R} \\ u^{\bar{\alpha}_{j}} B\left(\bar{\beta}_{j} \ln u\right) \tau_{j} & \text { se } \mu_{j} \notin \mathbb{R}\end{array} \quad j=2, \ldots, k\right.$, sendo $\bar{\alpha}_{j}=\frac{\alpha_{j}}{\alpha_{1}}$ e $\bar{\beta}_{j}=\frac{\beta_{j}}{\alpha_{1}}$.

Observe que, como $\alpha_{k}<\cdots<\alpha_{1}<0$, então $1<\frac{\alpha_{2}}{\alpha_{1}}<\frac{\alpha_{3}}{\alpha_{1}}<\ldots<\frac{\alpha_{k}}{\alpha_{1}}$, ou seja, $1<\overline{\alpha_{2}}<\overline{\alpha_{3}}<\ldots<\overline{\alpha_{k}}$.

Da equação 2.17 obtemos:

$f_{1}\left(X_{u}(\tau)\right)=a_{1} u+R_{1}\left(X_{u}(\tau)\right)$ e

a) se $\mu_{j} \in \mathbb{R}$ então $f_{j}\left(X_{u}(\tau)\right)=a_{j} u^{\bar{\alpha}_{j}} \tau_{j}+R_{j}\left(X_{u}(\tau)\right)$;

b) se $\mu_{j} \notin \mathbb{R}$ então $f_{j}\left(X_{u}(\tau)\right)=A_{j} u^{\bar{\alpha}_{j}} B\left(\bar{\beta}_{j} \ln u\right) \tau_{j}+R_{j}\left(X_{u}(\tau)\right)$. 
Por outro lado, como $f$ transforma a órbita de $X$ pelo ponto $\tau$ na órbita pelo ponto $\tilde{f}(\tau)$, obtemos de 2.19 que:

$\left.\mathrm{a}^{\prime}\right)$ se $\mu_{j} \in \mathbb{R}$ então $f_{j}\left(X_{u}(\tau)\right)=\left[f_{1}\left(X_{u}(\tau)\right)\right]^{\bar{\alpha}_{j}} \tilde{f}_{j}(\tau)$;

b') se $\mu_{j} \notin \mathbb{R}$ então $f_{j}\left(X_{u}(\tau)\right)=\left[f_{1}\left(X_{u}(\tau)\right)\right]^{\bar{\alpha}_{j}} B\left[\bar{\beta}_{j} \ln \left(f_{1}\left(X_{u}(\tau)\right)\right)\right] \tilde{f}_{j}(\tau)$.

O que faremos agora será comparar a) com a') e b) com b').

$\Rightarrow$ Comparando a) e a') temos:

$$
a_{j} u^{\bar{\alpha}_{j}} \tau_{j}+R_{j}\left(X_{u}(\tau)\right)=\left[f_{1}\left(X_{u}(\tau)\right)\right]^{\bar{\alpha}_{j}} \tilde{f}_{j}(\tau)
$$

ou ainda,

$$
a_{j} u^{\bar{\alpha}_{j}} \tau_{j}+R_{j}\left(X_{u}(\tau)\right)=\left[a_{1} u+R_{1}\left(X_{u}(\tau)\right)\right]^{\bar{\alpha}_{j}} \tilde{f}_{j}(\tau) .
$$

Multiplicando ambos os lados desta última igualdade por $u^{-\overline{\alpha_{j}}}$ obtemos:

$$
a_{j} \tau_{j}+u^{-\bar{\alpha}_{j}} R_{j}\left(X_{u}(\tau)\right)=\left[a_{1}+\frac{R_{1}\left(X_{u}(\tau)\right)}{u}\right]^{\bar{\alpha}_{j}} \tilde{f}_{j}(\tau) .
$$

Derivando 2.20 em relação a $\tau_{j}$ :

$$
\begin{aligned}
a_{j}+u^{-\bar{\alpha}_{j}} u^{\bar{\alpha}_{j}} D_{j} R_{j}\left(X_{u}(\tau)\right)= & \\
=\quad \bar{\alpha}_{j}\left[a_{1}+\frac{R_{1}\left(X_{u}(\tau)\right)}{u}\right]^{\bar{\alpha}_{j}-1} \frac{u^{\alpha_{j}}}{u} & D_{j} R_{1}\left(X_{u}(\tau)\right) \tilde{f}_{j}(\tau)+ \\
& +\left[a_{1}+\frac{R_{1}\left(X_{u}(\tau)\right)}{u}\right]^{\bar{\alpha}_{j}} \frac{\partial \tilde{f}_{j}}{\partial \tau_{j}}(\tau) .
\end{aligned}
$$

Obtemos então:

$$
\begin{aligned}
a_{j}+D_{j} R_{j}\left(X_{u}(\tau)\right) & = \\
=\quad \bar{\alpha}_{j}\left[a_{1} u+R_{1}\left(X_{u}(\tau)\right)\right]^{\alpha_{j}-1} & D_{j} R_{1}\left(X_{u}(\tau)\right) \tilde{f}_{j}(\tau)+ \\
+ & +\left[a_{1}+\frac{R_{1}\left(X_{u}(\tau)\right)}{u}\right]^{\bar{\alpha}_{j}} \frac{\partial \tilde{f}_{j}}{\partial \tau_{j}}(\tau) .
\end{aligned}
$$


Calcularemos agora o limite da expressão 2.21 quando $u \rightarrow 0$. De 2.17 temos que $D_{j} f_{j}(x)=a_{j}+D_{j} R_{j}(x)$. Além disso temos também que $\lim _{x \rightarrow 0} D_{j} f_{j}(x)=$ $=D_{j} f_{j}(0)=a_{j}$. Portanto,

$$
\lim _{u \rightarrow 0} D_{j} R_{j}\left(X_{u}(\tau)\right)=0
$$

Analogamente,

$$
\lim _{u \rightarrow 0} D_{j} R_{1}\left(X_{u}(\tau)\right)=0
$$

pois, para $j \neq 1, \quad D_{j} f_{1}(x)=D_{j} R_{1}(x)$ e $\lim _{x \rightarrow 0} D_{j} f_{1}(x)=D_{j} f_{1}(0)=0$.

Temos também que

$$
\lim _{u \rightarrow 0}\left[a_{1} u+R_{1}\left(X_{u}(\tau)\right)\right]^{\alpha_{j}-1}=0
$$

e

$$
\lim _{u \rightarrow 0} \frac{R_{1}\left(X_{u}(\tau)\right)}{u}=0
$$

visto que $\bar{\alpha}_{j}>1, \lim _{u \rightarrow 0} \frac{R_{1}\left(X_{u}(\tau)\right.}{\left\|X_{u}(\tau)\right\|}=0$ e $\frac{\left\|X_{u}(\tau)\right\|}{u}$ é limitado quando $u \rightarrow 0$.

Assim, fazendo $u \rightarrow 0$ em 2.21 e usando $2.22,2.23,2.24$ e 2.25 concluímos que $a_{j}=a_{1}^{\bar{\alpha}_{j}} \frac{\partial \tilde{f}_{j}}{\partial \tau_{j}}(\tau)$. Portanto,

$$
\frac{\partial \tilde{f}_{j}}{\partial \tau_{j}}(\tau)=a_{1}^{-\bar{\alpha}_{j}} a_{j}
$$

Obtemos então

$$
\tilde{f}_{j}(\tau)=a_{1}^{-\bar{\alpha}_{j}} a_{j} \tau_{j}+\varphi\left(\tau_{2}, \ldots, \tau_{j-1}, \tau_{j+1}, \ldots, \tau_{k}\right)
$$

Derivemos agora 2.20 com relação a $\tau_{l}, \quad l \neq j$. Temos dois casos a analisar: quando $\mu_{l} \in \mathbb{R}$ e quando $\mu_{l} \notin \mathbb{R}$. 
- Suponhamos que $\mu_{l} \in \mathbb{R}$. Então obtemos:

$$
\begin{aligned}
& u^{-\overline{\alpha_{j}}} u^{\bar{\alpha}_{l}} D_{l} R_{j}\left(X_{u}(\tau)\right)= \\
& =\bar{\alpha}_{j}\left[a_{1}+\frac{R_{1}\left(X_{u}(\tau)\right)}{u}\right]^{\overline{\alpha_{j}}-1} \frac{u^{\overline{\alpha_{l}}}}{u} D_{l} R_{1}\left(X_{u}(\tau)\right) \tilde{f}_{j}(\tau)+ \\
& +\left[a_{1}+\frac{R_{1}\left(X_{u}(\tau)\right)}{u}\right]^{\alpha_{j}} \frac{\partial \tilde{f}_{j}}{\partial \tau_{l}}(\tau)
\end{aligned}
$$

ou ainda,

$$
\begin{aligned}
u^{\alpha_{l}-\bar{\alpha}_{j}} D_{l} R_{j} & \left(X_{u}(\tau)\right)= \\
= & \bar{\alpha}_{j}\left[a_{1}+\frac{R_{1}\left(X_{u}(\tau)\right)}{u}\right]^{\alpha_{j}-1} \cdot u^{\alpha_{l}-1} D_{l} R_{1}\left(X_{u}(\tau)\right) \tilde{f}_{j}(\tau)+ \\
+ & {\left[a_{1}+\frac{R_{1}\left(X_{u}(\tau)\right)}{u}\right]^{\alpha_{j}} \frac{\partial \tilde{f}_{j}}{\partial \tau_{l}}(\tau) . }
\end{aligned}
$$

Sabendo que $1<\bar{\alpha}_{2}<\ldots<\bar{\alpha}_{k}$, fazendo $u \rightarrow 0$ nesta última expressão obtemos, para $l>j$ :

$$
\frac{\partial \tilde{f}_{j}}{\partial \tau_{l}}(\tau)=0
$$

De 2.26 e 2.27 concluímos que

$$
\tilde{f}_{j}(\tau)=a_{1}^{-\tilde{\alpha}_{j}} a_{j} \tau_{j}+\varphi\left(\tau_{2}, \ldots, \tau_{j-1}\right) \text {. }
$$

- Suponhamos agora que $\mu_{l} \notin \mathbb{R}$. Então, derivando 2.20 tem relação a $\tau_{l}$, segue que:

$$
\begin{aligned}
u^{\overline{\alpha_{l}}-\bar{\alpha}_{j}} B\left(\bar{\beta}_{l} \ln u\right) D_{l} R_{j}\left(X_{u}(\tau)\right)= & \\
=\bar{\alpha}_{j}\left[a_{1}+\frac{R_{1}\left(X_{u}(\tau)\right)}{u}\right]^{\overline{\alpha_{j}}-1} u^{\overline{\alpha_{l}}-1} B\left(\bar{\beta}_{l} \ln u\right) D_{l} R_{1}\left(X_{u}(\tau)\right) \tilde{f}_{j}(\tau)+ & +\left[a_{1}+\frac{R_{1}\left(X_{u}(\tau)\right)}{u}\right]^{\bar{\alpha}_{j}} \frac{\partial \tilde{f}_{j}}{\partial \tau_{l}}(\tau) . \\
& +
\end{aligned}
$$


Fazendo $u \rightarrow 0$ nesta última equação e usando raciocínio análogo ao anterior (tendo que $B\left(\bar{\beta}_{l} \ln u\right)$ é limitado) chegamos a mesma conclusão que em 2.28 .

$\Rightarrow$ Comparemos agora b) e b'), onde $\mu_{j} \notin \mathbb{R}$. Temos :

$$
A_{j} u^{\alpha_{j}} B\left(\bar{\beta}_{j} \ln u\right) \tau_{j}+R_{j}\left(X_{u}(\tau)\right)=\left[f_{1}\left(X_{u}(\tau)\right)\right]^{\alpha_{j}} B\left[\bar{\beta}_{j} \ln \left(f_{1}\left(X_{u}(\tau)\right)\right)\right] \tilde{f}_{j}(\tau)
$$

ou ainda,

$$
\begin{aligned}
A_{j} u^{\bar{\alpha}_{j}} B\left(\bar{\beta}_{j} \ln u\right) \tau_{j}+R_{j}\left(X_{u}(\tau)\right)= \\
=\left[a_{1} u+R_{1}\left(X_{u}(\tau)\right)\right]^{\bar{\alpha}_{j}} B\left[\bar{\beta}_{j} \ln \left(a_{1} u+R_{1}\left(X_{u}(\tau)\right)\right)\right] \tilde{f}_{j}(\tau) .
\end{aligned}
$$

Multiplicando ambos os membros por $u^{-\bar{\alpha}_{j}} B\left(-\bar{\beta}_{j} \ln u\right)$ :

$$
\begin{aligned}
A_{j} \tau_{j}+u^{-\alpha_{j}} B\left(-\bar{\beta}_{j} \ln u\right) R_{j}\left(X_{u}(\tau)\right)= \\
=\left[a_{1}+\frac{R_{1}\left(X_{u}(\tau)\right)}{u}\right]^{\alpha_{j}} B\left[\bar{\beta}_{j} \ln \left(a_{1}+\frac{R_{1}\left(X_{u}(\tau)\right)}{u}\right)\right] \tilde{f}_{j}(\tau) .
\end{aligned}
$$

Derivando 2.29 com relação a $\tau_{j}$ obtemos:

$$
\begin{aligned}
A_{j}+u^{-\alpha_{j}} B\left(-\bar{\beta}_{j} \ln u\right) u^{\alpha_{j}} B\left(\vec{\beta}_{j} \ln u\right) D_{j} R_{j}\left(X_{u}(\tau)\right)= \\
=\bar{\alpha}_{j}\left[a_{1}+\frac{R_{1}\left(X_{u}(\tau)\right)}{u}\right]^{\bar{\alpha}_{j}-1} B\left(\bar{\beta}_{j} \ln u\right) \frac{u^{\alpha_{j}}}{u} D_{j} R_{1}\left(X_{u}(\tau)\right) \\
\cdot B\left[\bar{\beta}_{j} \ln \left(a_{1}+\frac{R_{1}\left(X_{u}(\tau)\right)}{u}\right)\right] \tilde{f}_{j}(\tau)+ \\
+\left[a_{1}+\frac{R_{1}\left(X_{u}(\tau)\right)}{u}\right]^{\alpha_{j}} D_{j} B\left[\bar{\beta}_{j} \ln \left(a_{1}+\frac{R_{1}\left(X_{u}(\tau)\right)}{u}\right)\right] \tilde{f}_{j}(\tau)+ \\
+\left[a_{1}+\frac{R_{1}\left(X_{u}(\tau)\right)}{u}\right]^{\bar{\alpha}_{j}} B\left[\bar{\beta}_{j} \ln \left(a_{1}+\frac{R_{1}\left(X_{u}(\tau)\right)}{u}\right)\right] \frac{\partial \tilde{f}_{j}}{\partial \tau_{j}}(\tau)
\end{aligned}
$$


onde

$$
\begin{aligned}
& D_{j} B\left[\bar{\beta}_{j} \ln \left(a_{1}+\frac{R_{1}\left(X_{u}(\tau)\right)}{u}\right)\right]= \\
&=\bar{\beta}_{j} \frac{1}{\left[a_{1}+\frac{R_{1}\left(X_{u}(\tau)\right)}{u}\right]} B\left(\bar{\beta}_{j} \ln u\right) D_{j} R_{1}\left(X_{u}(\tau)\right) \frac{u^{\bar{\alpha}_{j}}}{u} . \\
& \cdot \bar{B}\left[\bar{\beta}_{j} \ln \left(a_{1}+\frac{R_{1}\left(X_{u}(\tau)\right)}{u}\right)\right],
\end{aligned}
$$

$\operatorname{com} \bar{B}(t)=\left(\begin{array}{rr}-\operatorname{sen} t & \cos t \\ -\cos t & -\operatorname{sen} t\end{array}\right)$.

Simplificando:

$$
\begin{aligned}
& A_{j}+D_{j} R_{j}\left(X_{u}(\tau)\right)=\left[a_{1} u+R_{1}\left(X_{u}(\tau)\right)\right]^{\bar{\alpha}_{j}-1} \tilde{f}_{j}(\tau) D_{j} R_{1}\left(X_{u}(\tau)\right) \cdot \\
& \cdot\left\{\bar{\alpha}_{j} B\left[\bar{\beta}_{j} \ln \left(a_{1} u+R_{1}\left(X_{u}(\tau)\right)\right)\right]+\bar{\beta}_{j} B\left(\bar{\beta}_{j} \ln u\right) \bar{B}\left[\bar{\beta}_{j} \ln \left(a_{1}+\frac{R_{1}\left(X_{u}(\tau)\right)}{u}\right)\right]\right\}+ \\
& +\left[a_{1}+\frac{R_{1}\left(X_{u}(\tau)\right)}{u}\right]^{\bar{\alpha}_{j}} B\left[\bar{\beta}_{j} \ln \left(a_{1}+\frac{R_{1}\left(X_{u}(\tau)\right)}{u}\right)\right] \frac{\partial \tilde{f}_{j}}{\partial \tau_{j}}(\tau),
\end{aligned}
$$

ou ainda,

$$
\begin{gathered}
A_{j}+D_{j} R_{j}\left(X_{u}(\tau)\right)=\left[a_{1} u+R_{1}\left(X_{u}(\tau)\right)\right]^{\bar{\alpha}_{j}-1} \tilde{f}_{j}(\tau) D_{j} R_{1}\left(X_{u}(\tau)\right) . \\
\cdot\left[\begin{array}{cc}
\bar{\alpha}_{j} & \bar{\beta}_{j} \\
-\bar{\beta}_{j} & \bar{\alpha}_{j}
\end{array}\right] B\left[\bar{\beta}_{j} \ln \left(a_{1} u+R_{1}\left(X_{u}(\tau)\right)\right)\right]+ \\
+\left[a_{1}+\frac{R_{1}\left(X_{u}(\tau)\right)}{u}\right]^{\bar{\alpha}_{j}} B\left[\bar{\beta}_{j} \ln \left(a_{1}+\frac{R_{1}\left(X_{u}(\tau)\right)}{u}\right)\right] \frac{\partial \tilde{f}_{j}}{\partial \tau_{j}}(\tau) .
\end{gathered}
$$

Calculemos agora o limite da expressão 2.30 quando $u \rightarrow 0$. Por um mesmo raciocínio anterior temos que 


$$
\begin{aligned}
& \lim _{u \rightarrow 0} D_{j} R_{j}\left(X_{u}(\tau)\right)=0 ; \\
& \lim _{u \rightarrow 0} D_{j} R_{1}\left(X_{u}(\tau)\right)=0 ; \\
& \lim _{u \rightarrow 0}\left[a_{1} u+R_{1}\left(X_{u}(\tau)\right)\right]^{\alpha_{j}-1}=0 \mathrm{e} \\
& \lim _{u \rightarrow 0}\left[a_{1}+\frac{R_{1}\left(X_{u}(\tau)\right)}{u}\right]^{\alpha_{j}}=a_{1}^{\alpha_{j}} .
\end{aligned}
$$

Além disso, temos também que

$$
B\left[\bar{\beta}_{j} \ln \left(a_{1} u+R_{1}\left(X_{u}(\tau)\right)\right)\right] \text { é limitado. }
$$

Consequentemente, fazendo $u \rightarrow 0 \mathrm{em} 2.30$ e usando os resultados acima, obtemos

ou ainda,

$$
A_{j}=a_{1}^{\bar{\alpha}_{j}} B\left(\bar{\rho}_{j} \ln a_{1}\right) \frac{\partial \tilde{f}_{j}}{\partial \tau_{j}}(\tau)
$$

$$
\frac{\partial \tilde{f}_{j}}{\partial \tau_{j}}(\tau)=a_{1}^{-\bar{\alpha}_{j}} A_{j} B\left(-\bar{\beta}_{j} \ln a_{1}\right)
$$

Obtemos então:

$$
\tilde{f}_{j}(\tau)=a_{1}^{-\bar{\alpha}_{j}} A_{j} B\left(-\bar{\beta}_{j} \ln a_{1}\right) \tau_{j}+\varphi\left(\tau_{2}, \ldots, \tau_{k}\right) .
$$

Derivemos agora 2.29 com relação a $\tau_{l}, \quad l \neq j$. Novamente teremos dois casos a analisar: quando $\mu_{l} \in \mathbb{R}$ e quando $\mu_{l} \notin \mathbb{R}$. Faremos apenas um dos casos, visto que o outro é análogo. Assim, supondo que $\mu_{l} \in \mathbb{R}$, obtemos:

$$
\begin{aligned}
& u^{-\bar{\alpha}_{j}} B\left(-\bar{\beta}_{j} \ln u\right) u^{\bar{\alpha}_{l}} D_{l} R_{j}\left(X_{u}(\tau)\right)= \\
& =\bar{\alpha}_{j}\left[a_{1}+\frac{R_{1}\left(X_{u}(\tau)\right)}{u}\right]^{\bar{\alpha}_{j}-1} \frac{u^{\bar{\alpha}_{1}}}{u} D_{l} R_{1}\left(X_{u}(\tau)\right) . \\
& \cdot B\left[\bar{\beta}_{j} \ln \left(a_{1}+\frac{R_{1}\left(X_{u}(\tau)\right)}{u}\right)\right] \tilde{f}_{j}(\tau)+ \\
& +\left[a_{1}+\frac{R_{1}\left(X_{u}(\tau)\right)}{u}\right]^{\bar{\alpha}_{j}} D_{l} B\left[\bar{\beta}_{j} \ln \left(a_{1}+\frac{R_{1}\left(X_{u}(\tau)\right)}{u}\right)\right] \tilde{f}_{j}(\tau)+ \\
& +\left[a_{1}+\frac{R_{1}\left(X_{u}(\tau)\right)}{u}\right]^{\alpha_{j}} B\left[\bar{\beta}_{j} \ln \left(a_{1}+\frac{R_{1}\left(X_{u}(\tau)\right)}{u}\right)\right] \frac{\partial \tilde{f}_{j}}{\partial n}(\tau),
\end{aligned}
$$


onde,

$$
\begin{aligned}
D_{l} B\left[\bar{\beta}_{j} \ln \left(a_{1}+\frac{R_{1}\left(X_{u}(\tau)\right)}{u}\right)\right]= \\
=\bar{\beta}_{j} \frac{1}{\left[a_{1}+\frac{R_{1}\left(X_{u}(\tau)\right)}{u}\right]} D_{l} R_{1}\left(X_{u}(\tau)\right) \frac{u^{\bar{\alpha}_{l}}}{u} \\
\cdot \bar{B}\left[\bar{\beta}_{j} \ln \left(a_{1}+\frac{R_{1}\left(X_{u}(\tau)\right)}{u}\right)\right],
\end{aligned}
$$

$\operatorname{com} \bar{B}(t)=\left(\begin{array}{rr}-\operatorname{sen} t & \cos t \\ -\cos t & -\operatorname{sen} t\end{array}\right)$.

Reescrevendo a última equação, temos:

$$
\begin{aligned}
& u^{\bar{\alpha}_{l}-\bar{\alpha}_{j}} B\left(-\bar{\beta}_{j} \ln u\right) D_{l} R_{j}\left(X_{u}(\tau)\right)= \\
& =\left[a_{1}+\frac{R_{1}\left(X_{u}(\tau)\right)}{u}\right]^{\alpha_{j}-1} \tilde{f}_{j}(\tau) D_{l} R_{1}\left(X_{u}(\tau)\right) u^{\alpha_{l-1}} . \\
& \cdot\left\{\bar{\alpha}_{j} B\left[\bar{\beta}_{j} \ln \left(a_{1}+\frac{R_{1}\left(X_{u}(\tau)\right)}{u}\right)\right]+\bar{\beta}_{j} \bar{B}\left[\bar{\beta}_{j} \ln \left(a_{1}+\frac{R_{1}\left(X_{u}(\tau)\right)}{u}\right)\right]\right\}+ \\
& +\left[a_{1}+\frac{R_{1}\left(X_{u}(\tau)\right)}{u}\right]^{\bar{\alpha}_{j}} B\left[\bar{\beta}_{j} \ln \left(a_{1}+\frac{R_{1}\left(X_{u}(\tau)\right)}{u}\right)\right] \frac{\partial \tilde{f}_{j}}{\partial \tau_{l}}(\tau) .
\end{aligned}
$$

Simplificando:

$$
\begin{aligned}
u^{\bar{\alpha}_{l}-\bar{\alpha}_{j}} B\left(-\bar{\beta}_{j} \ln u\right) & D_{l} R_{j}\left(X_{u}(\tau)\right)= \\
= & {\left[a_{1}+\frac{R_{1}\left(X_{u}(\tau)\right)}{u}\right]^{\bar{\alpha}_{j}-1} \tilde{f}_{j}(\tau) D_{l} R_{1}\left(X_{u}(\tau)\right) u^{\bar{\alpha}_{l-1}} . } \\
& \cdot\left[\begin{array}{cc}
\bar{\alpha}_{j} & \bar{\beta}_{j} \\
-\bar{\beta}_{j} & \bar{\alpha}_{j}
\end{array}\right] B\left[\bar{\beta}_{j} \ln \left(a_{1}+\frac{R_{1}\left(X_{u}(\tau)\right)}{u}\right)\right]+ \\
& +\left[a_{1}+\frac{R_{1}\left(X_{u}(\tau)\right)}{u}\right]^{\alpha_{j}} B\left[\bar{\beta}_{j} \ln \left(a_{1}+\frac{R_{1}\left(X_{u}(\tau)\right)}{u}\right)\right] \frac{\partial \tilde{f}_{j}}{\partial \tau_{l}}(\tau) .
\end{aligned}
$$

Fazendo $u \rightarrow 0$ nesta última expressão, com o mesmo raciocínio feito anteriormente, concluímos que, para $l>j$ :

$$
\frac{\partial \tilde{f}_{j}}{\partial \tau_{l}}(\tau)=0
$$


De 2.31 e 2.32 conclúmos que

$$
\tilde{f}_{j}(\tau)=a_{1}^{-\bar{\alpha}_{j}} A_{j} B\left(-\bar{\beta}_{j} \ln a_{1}\right) \tau_{j}+\varphi\left(\tau_{2}, \cdots, \tau_{j-1}\right) \text {. }
$$

Das equações 2.28 e 2.33 conclui-se a parte $\mathrm{A}$ do Teorema 2.1 para o caso em que $a_{1}>0$. Observe que a condição $\alpha_{k}<\cdots<\alpha_{1}<0$ foi fundamental para esta conclusão.

Supondo agora que $a_{1}=\frac{\partial f_{1}}{\partial x_{1}}(0)<0$ então $\tilde{f}^{2}\left(\Sigma_{1}\right) \subset \Sigma_{1}$ e $\tilde{f}^{2}\left(\Sigma_{-1}\right) \subset \Sigma_{-1}$. Deste modo as mesmas conclusões do caso anterior são válidas para $\tilde{f}^{2}$, visto que $f^{2}$ com $a_{1}<0$ está nas mesmas condições que $f \operatorname{com} a_{1}>0$.

CASO B: Suponhamos que $\beta_{1} \neq 0$. Neste caso consideremos o seguinte conjunto de autovalores do campo $X$ :

$$
\left\{\begin{array}{l}
\mu_{1}=\alpha_{1} \pm i \beta_{1} \\
\mu_{2}=\alpha_{2} \pm i \beta_{2} \\
\vdots \\
\mu_{k}=\alpha_{k} \pm i \beta_{k}
\end{array}\right.
$$

$k \leq n, \operatorname{com} \alpha_{k}<\ldots<\alpha_{1}<0$, onde o autoespaço $E_{1}$ associado a $\mu_{1}$ tem dimensão 2 .

Analogamente ao que foi feito no caso $\mathrm{A}$, escrevemos $f: U \subset \mathbb{R}^{n} \rightarrow \mathbb{R}^{n}$ como $f=\left(f_{1}, \ldots, f_{k}\right)$, onde $f_{j}: U \subset \mathbb{R}^{n} \rightarrow E_{j}, \quad j=1, \ldots, k$ é dada por:

$$
f_{j}\left(x_{1}, \ldots, x_{k}\right)=A_{j} x_{j}+R_{j}\left(x_{1}, \ldots, x_{k}\right)
$$

sendo

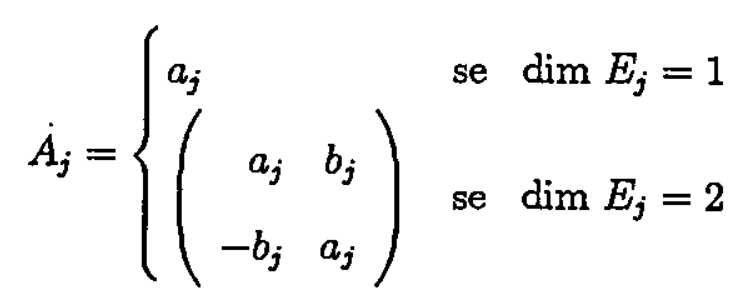


e $\lim _{x \rightarrow 0} \frac{R_{j}(x)}{\|x\|}=0, \quad j=1, \ldots, k, \operatorname{com} a_{j}, b_{j} \in \mathbb{R}$

Como $\Sigma=S^{1} \times E$, sendo $E=E_{2} \times \cdots E_{k}$ e $\tilde{f}: \Sigma \longrightarrow \Sigma$, denotaremas $\tau=\left(\tau_{1}, \tau_{2}, \ldots, \tau_{k}\right) \in \Sigma$ por $\left(\cos \theta, \operatorname{sen} \theta, \tau_{2}, \ldots, \tau_{k}\right)$ e $\left(\tilde{f}_{1}(\tau), \ldots, \tilde{f}_{k}(\tau)\right) \in \Sigma$ por $\left(\tilde{f}_{\theta}(\tau), \tilde{f}_{2}(\tau), \ldots, \tilde{f}_{k}(\tau)\right)$

A trajetória de $X$ pelo ponto $\tau \in \Sigma$ é dada por:

$$
X_{t}(\tau)=\left(X_{t}^{1}(\tau), \cdots, X_{t}^{j}(\tau), \cdots, X_{t}^{k}(\tau)\right)
$$

onde

$$
X_{t}^{1}(\tau)=e^{\alpha_{1} t} B\left(\beta_{1} t\right) \tau_{1} \quad \ominus X_{t}^{j}(\tau)=\left\{\begin{array}{ll}
e^{\alpha_{j} t} \tau_{j} & \text { se } \mu_{j} \in \mathbb{R} \\
e^{\alpha_{j} t} B\left(\beta_{j} t\right) \tau_{j} & \text { se } \mu_{j} \notin \mathbb{R}
\end{array} \quad j=2, \ldots, k\right.
$$

$\operatorname{com} B(t)=\left(\begin{array}{rr}\cos t & \operatorname{sen} t \\ -\operatorname{sen} t & \cos t\end{array}\right)$

Para encontrarmos uma expressão para $\tilde{f}$, faremos o mesmo raciocínio anterior, ou seja, reescreveremos a órbita do campo $X$ em função de um novo parâmetro.

Seja $r=\left\|X_{t}^{1}(\tau)\right\|$. Então $r=e^{\alpha_{1} t}$. Façamos $t=\frac{1}{\alpha_{1}} \ln r$. Assim,

$$
X_{r}(\tau)=\left(X_{r}^{1}(\tau), \cdots, X_{r}^{j}(\tau), \cdots, X_{r}^{k}(\tau)\right)
$$

onde,

$$
X_{r}^{1}(\tau)=r B\left(\bar{\beta}_{1} \ln r\right) \tau_{1} \quad \text { e } \quad X_{r}^{j}(\tau)=\left\{\begin{array}{ll}
r^{\bar{\alpha}_{j}} \tau_{j} & \text { se } \mu_{j} \in \mathbb{R} \\
r^{\bar{\alpha}_{j}} B\left(\bar{\beta}_{j} \ln r\right) \tau_{j} & \text { se } \mu_{j} \notin \mathbb{R}
\end{array} \quad j=2, \ldots, k\right.
$$

sendo $\tau_{1}=(\cos \theta, \operatorname{sen} \theta), \bar{\alpha}_{j}=\frac{\alpha_{j}}{\alpha_{1}}$ e $\bar{\beta}_{j}=\frac{\beta_{j}}{\alpha_{1}}$. 
De 2.34 obtemos:

$$
\begin{aligned}
& f_{1}\left(X_{r}(\tau)\right)=A_{1} r B\left(\bar{\beta}_{1} \ln r\right) \tau_{1}+R_{1}\left(X_{r}(\tau)\right), \\
& f_{j}\left(X_{r}(\tau)\right)=\left\{\begin{array}{ll}
a_{j} r^{\overline{\alpha_{j}}} \tau_{j}+R_{j}\left(X_{\tau}(\tau)\right) & \text { se } \mu_{j} \in \mathbb{R} \\
A_{j} r^{\alpha_{j}} B\left(\bar{\beta}_{j} \ln r\right) \tau_{j}+R_{j}\left(X_{\tau}(\tau)\right) & \text { se } \mu_{j} \notin \mathbb{R}
\end{array}, j=2, \ldots, k .\right.
\end{aligned}
$$

Por outro lado, como $f$ transforma a órbita do campo $X$ pelo ponto $\tau$ na órbita pelo ponto $\tilde{f}(\tau)$, usando 2.35 temos também que:

$$
\begin{aligned}
& f_{1}\left(X_{r}(\tau)\right)=\left\|f_{1}\left(X_{r}(\tau)\right)\right\| B\left[\bar{\beta}_{1} \ln \left(\left\|f_{1}\left(X_{r}(\tau)\right)\right\|\right)\right] \tilde{f}_{\theta}(\tau), \\
& f_{j}\left(X_{r}(\tau)\right)= \begin{cases}\left\|f_{1}\left(X_{r}(\tau)\right)\right\|^{\bar{\alpha}_{j}} \tilde{f}_{j}(\tau) & \text { se } \mu_{j} \in \mathbb{R} \\
\left\|f_{1}\left(X_{r}(\tau)\right)\right\|^{\bar{\alpha}_{j}} B\left[\bar{\beta}_{j} \ln \left(\left\|f_{1}\left(X_{r}(\tau)\right)\right\|\right)\right] \tilde{f}_{j}(\tau) & \text { se } \mu_{j} \notin \mathbb{R},\end{cases}
\end{aligned}
$$

onde $j=2, \ldots, k$.

Encontremos então $\left\|f_{1}(x)\right\|$. Como $\operatorname{dim} E_{1}=2$, temos $f_{1}=\left(f_{11}, f_{12}\right)$. Seja $\rho(x)=\left\|f_{1}(x)\right\|$. De 2.34 temos que $f_{1}(x)=A_{1} x_{1}+R_{1}(x)$, onde $x=\left(x_{1}, \ldots, x_{k}\right)$, $\lim _{x \rightarrow 0} \frac{R_{1}(x)}{\|x\|}=0$ e $A_{1}=\left(\begin{array}{cc}a_{1} & b_{1} \\ -b_{1} & a_{1}\end{array}\right)$. Escrevemos $\rho(x)=\left\|A_{1} x_{1}\right\|+\left\|A_{1} x_{1}+R_{1}(x)\right\|-$ $\left\|A_{1} x_{1}\right\|$. Seja $\bar{R}(x)=\left\|A_{1} x_{1}+R_{1}(x)\right\|-\left\|A_{1} x_{1}\right\|$. É fácil ver que $\lim _{x \rightarrow 0} \frac{\bar{R}(x)}{\|x\|}=0$. Assim, $\rho(x)=\left\|A_{1} x_{1}\right\|+\bar{R}(x)$. Temos então que

$$
\rho\left(X_{r}(\tau)\right)=\left\|A_{1} X_{r}^{1}(\tau)\right\|+\bar{R}\left(X_{r}(\tau)\right)=\delta r+\bar{R}\left(X_{r}(\tau)\right)
$$

onde $\delta=\sqrt{a^{2}+b^{2}}$ e $\lim _{x \rightarrow 0} \frac{\bar{R}(x)}{\|x\|}=0$.

Substituindo esta última expressão em 2.38 e 2.39, obtemos: 


$$
\begin{aligned}
f_{1}\left(X_{r}(\tau)\right) & =\left[\delta r+\bar{R}\left(X_{r}(\tau)\right)\right] B\left[\bar{\beta}_{1} \ln \left(\delta r+\bar{R}\left(X_{\mathrm{r}}(\tau)\right)\right)\right] \tilde{f}_{\theta}(\tau), \\
f_{j}\left(X_{\mathrm{r}}(\tau)\right) & = \begin{cases}{\left[\delta r+\bar{R}\left(X_{\mathrm{r}}(\tau)\right)\right]^{\alpha_{j}} \tilde{f}_{j}(\tau)} & \text { se } \mu_{j} \in \mathbb{R} \\
{\left[\delta r+\bar{R}\left(X_{r}(\tau)\right)\right]^{\alpha_{j}} B\left[\bar{\beta}_{j} \ln \left(\delta r+\bar{R}\left(X_{\mathrm{r}}(\tau)\right)\right)\right] \tilde{f}_{j}(\tau)} & \text { se } \mu_{j} \notin \mathbb{R},\end{cases}
\end{aligned}
$$

$2 \leq j \leq k$.

Comparando as equações 2.36 e 2.40 :

$$
A_{1} r B\left(\bar{\beta}_{1} \ln r\right) \tau_{1}+R_{1}\left(X_{r}(\tau)\right)=\left[\delta r+\bar{R}\left(X_{r}(\tau)\right)\right] B\left[\bar{\beta}_{1} \ln \left(\delta r+\vec{R}\left(X_{r}(\tau)\right)\right)\right] \tilde{f}_{\theta}(\tau)
$$

Multiplicando por $r^{-1} B\left(-\bar{\beta}_{1} \ln r\right)$ obtemos:

$$
\begin{aligned}
A_{1} \tau_{1}+B\left(-\bar{\beta}_{1} \ln r\right) \frac{R_{1}\left(X_{r}(\tau)\right.}{r} & = \\
= & {\left[\delta+\frac{\bar{R}\left(X_{r}(\tau)\right)}{r}\right] B\left[\bar{\beta}_{1} \ln \left(\delta+\frac{\bar{R}\left(X_{\mathrm{r}}(\tau)\right)}{r}\right)\right] \tilde{f}_{\theta}(\tau) . }
\end{aligned}
$$

Fazendo $r \rightarrow 0$ nesta última expressão e tendo que

$$
\lim _{r \rightarrow 0} \frac{\bar{R}\left(X_{r}(\tau)\right)}{r}=0=\lim _{r \rightarrow 0} \frac{R_{1}\left(X_{r}(\tau)\right)}{r}
$$

segue que

$$
A_{1} \tau_{1}=\delta B\left(\bar{\beta}_{1} \ln \delta\right) \tilde{f}_{\theta}(\tau)
$$

Portanto,

$$
\tilde{f}_{\theta}(\tau)=\delta^{-1} B\left(-\bar{\beta}_{1} \ln \delta\right) A_{1} \tau_{1}
$$

É fácil ver que $\delta^{-1} B\left(-\bar{\beta}_{1} \ln \delta\right) A_{1} \tau_{1}$ é uma rotação. Assim, escrevemos:

$$
\tilde{f}_{\theta}\left(\theta_{0}\right)=\theta_{0}+\varphi_{0} \text {, }
$$

onde $\varphi_{0}$ é constante. 
Analisaremos agora $\tilde{f}_{j}(x), \quad j=2, \ldots, k$. Comparando as equaçōes 2.37 e 2.41, temos dois casos a analisar: quando $\mu_{j} \in \mathbb{R}$ e quando $\mu_{j} \notin \mathbb{R}$. Analisaremos suscintamente ambos os casos.

- Suponhamos que $\mu_{j} \in \mathbb{R}$. Temos então

$$
a_{j} r^{\alpha_{j}} \tau_{j}+R_{j}\left(X_{r}(\tau)\right)=\left[\delta r+\bar{R}\left(X_{r}(\tau)\right)\right]^{\alpha_{j}} \tilde{f}_{j}(\tau)
$$

Multiplicando por $r^{-\alpha_{j}}$ :

$$
a_{j} \tau_{j}+r^{-\alpha_{j}} R_{j}\left(X_{r}(\tau)\right)=\left[\delta+\frac{\vec{R}\left(X_{r}(\tau)\right)}{r}\right]^{\alpha_{j}} \tilde{f}_{j}(\tau) .
$$

Derivando a equação 2.43 em relação a $\tau_{j}$ segue que

$$
\begin{aligned}
& a_{j}+r^{-\bar{\alpha}_{j}} r^{\alpha_{j}} D_{j} R_{j}\left(X_{r}(\tau)\right)== \\
&=\quad \bar{\alpha}_{j}\left[\delta+\frac{\bar{R}\left(X_{r}(\tau)\right)}{r}\right]^{\alpha_{j}-1} \frac{r^{\alpha_{j}}}{r} D_{j} \vec{R}\left(X_{r}(\tau)\right) \tilde{f}_{j}(\tau)+ \\
&+\left[\delta+\frac{\bar{R}\left(X_{r}(\tau)\right)}{r}\right]^{\alpha_{j}} \frac{\partial \tilde{f}_{j}}{\partial r_{j}}(\tau)
\end{aligned}
$$

ou ainda,

$$
\begin{aligned}
a_{j}+D_{j} R_{j}\left(X_{r}(\tau)\right) & = \\
=\quad & \bar{\alpha}_{j}\left[\delta r+\bar{R}\left(X_{r}(\tau)\right)\right]^{\alpha_{j}-1} D_{j} \tilde{R}\left(X_{r}(\tau)\right) \tilde{f}_{j}(\tau)+ \\
& +\left[\delta+\frac{\bar{R}\left(X_{r}(\tau)\right)}{r}\right]^{\alpha_{j}} \frac{\partial \tilde{f}_{j}}{\partial \tau_{j}}(\tau) .
\end{aligned}
$$

Fazendo $r \rightarrow 0$ e sabendo que

$$
\begin{aligned}
& \lim _{r \rightarrow 0} D_{j} \bar{R}\left(X_{r}(\tau)\right)=\lim _{r \rightarrow 0} D_{j} R_{j}\left(X_{r}(\tau)\right)=0 ; \\
& \lim _{r \rightarrow 0}\left[\delta r+\vec{R}\left(X_{r}(\tau)\right)\right]^{\bar{\alpha}_{j}-1}=0 \mathrm{e} \\
& \lim _{r \rightarrow 0}\left[\delta+\frac{\bar{R}\left(X_{r}(\tau)\right)}{r}\right]^{\alpha_{j}}=\delta^{\bar{\alpha}_{j}}
\end{aligned}
$$


obtemos

$$
a_{j}=\delta^{\bar{\alpha}_{j}} \frac{\partial \tilde{f}_{j}}{\partial \tau_{j}}(\tau)
$$

ou ainda,

$$
\frac{\partial \tilde{f}_{j}}{\partial \tau_{j}}(\tau)=\delta^{-\alpha_{j}} a_{j}
$$

Portanto

$$
\tilde{f}_{j}(\tau)=\delta^{-\bar{\alpha}_{j}} a_{j} \tau_{j}+\varphi\left(\tau_{1}, \ldots, \tau_{j-1}, \tau_{j+1}, \ldots, \tau_{k}\right)
$$

Derivemos agora 2.43 com relação a $\tau_{l}, \quad l \neq j$. Temos dois casos a analisar: quando $\mu_{l} \in \mathbb{R}$ e quando $\mu_{l} \notin \mathbb{R}$. Analisaremos apenas um deles, visto que o outro é análogo ao que foi feito anteriormente. Vamos supor que $\mu_{l} \in \mathbb{R}$. Então,

$$
\begin{aligned}
& r^{-\overline{\alpha_{j}}} r^{\overline{\alpha_{l}}} D_{l} R_{j}\left(X_{r}(\tau)\right)= \\
& =\bar{\alpha}_{j}\left[\delta+\frac{\vec{R}\left(X_{r}(\tau)\right)}{r}\right]^{\overline{\alpha_{j}-1}} \frac{r^{\overline{\alpha_{l}}}}{r} D_{l} \bar{R}\left(X_{r}(\tau)\right) \tilde{f}_{j}(\tau)+ \\
& +\left[\delta+\frac{\bar{R}\left(X_{r}(\tau)\right)}{r}\right]^{\alpha_{j}} \frac{\partial \tilde{f}_{j}}{\partial \tau_{l}}(\tau)
\end{aligned}
$$

ou ainda,

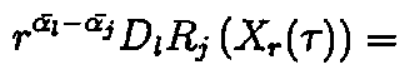

$$
\begin{aligned}
& =\bar{\alpha}_{j}\left[\delta+\frac{\bar{R}\left(X_{r}(\tau)\right)}{r}\right]^{\bar{\alpha}_{j}-1} r^{\bar{\alpha}_{l}-1} D_{l} \bar{R}\left(X_{r}(\tau)\right) \tilde{f}_{j}(\tau)+ \\
& +\left[\delta+\frac{\bar{R}\left(X_{r}(\tau)\right)}{r}\right]^{\alpha_{j}} \frac{\partial \tilde{f}_{j}}{\partial \tau_{l}}(\tau)
\end{aligned}
$$

Sabendo que $1<\vec{\alpha}_{2}<\ldots<\bar{\alpha}_{k}$ fazendo $r \rightarrow 0$ obtemos, para $l>j$

$$
\frac{\partial \tilde{f}_{j}}{\partial \tau_{l}}(\tau)=0
$$

Desta última expressão e da equação 2.44 segue que

$$
\tilde{f}_{j}(\tau)=\delta^{-\bar{\alpha}_{j}} a_{j} \tau_{j}+\varphi\left(\tau_{1}, \ldots, \tau_{j-1}\right)
$$

$\operatorname{com} j=2, \ldots, j$ e $A_{j} \in \mathbb{R}$. 
- Comparando as equações 2.37 e 2.41 , suponhamos agora que $\mu_{j} \notin \mathbb{R}$. Temos:

$$
A_{j} r^{\alpha_{j}} B\left(\bar{\beta}_{j} \ln r\right) \tau_{j}+R_{j}\left(X_{r}(\tau)\right)=\left[\delta r+\bar{R}\left(X_{r}(\tau)\right)\right]^{\bar{\alpha}_{j}} B\left[\bar{\beta}_{j} \ln \left(\delta r+\bar{R}\left(X_{r}(\tau)\right)\right)\right] \tilde{f}_{j}(\tau)
$$

Multiplicando por $r^{-\bar{\alpha}_{j}} B\left(-\bar{\beta}_{j} \ln r\right) \tau_{j}$ obtemos:

$$
\begin{aligned}
A_{j} \tau_{j}+r^{-\alpha_{j}} B\left(-\bar{\beta}_{j} \ln r\right) \tau_{j} R_{j}\left(X_{\tau}(\tau)\right)= \\
=\left[\delta+\frac{\vec{R}\left(X_{r}(\tau)\right)}{r}\right]^{\alpha_{j}} B\left[\bar{\beta}_{j} \ln \left(\delta+\frac{\bar{R}\left(X_{r}(\tau)\right)}{r}\right)\right] \tilde{f}_{j}(\tau) .
\end{aligned}
$$

Fazendo $r \rightarrow 0$ nesta última expressão, com um raciocínio análogo ao feito antes segue que

$$
\tilde{f}_{j}(\tau)=\delta^{-\alpha_{j}} A_{j} \tau_{j}+\varphi\left(\tau_{1}, \ldots, \tau_{j-1}\right) \text {, }
$$

$\operatorname{com} j=2, \ldots, j$ e $A_{j}=\left(\begin{array}{rr}a_{j} & b_{j} \\ -b_{j} & a_{j}\end{array}\right)$

As equações $2.42,2.45$ e 2.47 constituem exatamente a conclusão da parte B do Teorema 2.1.

\subsection{Prova do Teorema 2.2}

Passemos agora para a prova do Teorema 2.2, cujas hipóteses são, além daquelas do Teorema 2.1, que os autovalores do campo $X$ satisfazem às condições de não ressonância e também que $f \in C^{l}, l>\bar{\alpha}_{k}$. Portanto as conclusões do Teorema 2.1 são válidas.

\section{Demonstraçāo:}

Como antes, temos $f_{j}(x)=A_{j} x_{j}+R_{j}(x), j=1, \ldots, k$. Como $f \in C^{l}$, podemos escrever $R_{j}(x)=P_{j}(x)+Q_{j}(x)$, onde $P_{j}(x)$ é um polinômio de grau maior ou igual 
a 2 e menor ou igual a $l$ e

$$
\lim _{x \rightarrow 0} \frac{P_{j}(x)}{\|x\|}=0 \quad \text { e } \quad \lim _{x \rightarrow 0} \frac{Q_{j}(x)}{\|x\|^{l}}=0 .
$$

Como na prova do Teorema 2.1, temos dois casos para analisar: quando $\beta_{1}=0$ e quando $\beta_{1} \neq 0$.

CASO A-Suponhamos que $\beta_{1}=0$. Neste caso, das equações 2.20 e 2.29 temos, respectivamente:

-se $\mu_{j} \in \mathbb{R}$ :

$$
a_{j} \tau_{j}+u^{-\bar{\alpha}_{j}} R_{j}\left(X_{u}(\tau)\right)=\left[a_{1}+\frac{R_{1}\left(X_{u}(\tau)\right)}{u}\right]^{\alpha_{j}} \tilde{f}_{j}(\tau)
$$

-se $\mu_{j} \notin \mathbb{R}:$

$$
\begin{aligned}
A_{j} \tau_{j}+u^{-\bar{\alpha}_{j}} B\left(-\bar{\beta}_{j} \ln u\right) & R_{j}\left(X_{u}(\tau)\right)= \\
= & {\left[a_{1}+\frac{R_{1}\left(X_{u}(\tau)\right)}{u}\right]^{\alpha_{j}} B\left[\bar{\beta}_{j} \ln \left(a_{1}+\frac{R_{1}\left(X_{u}(\tau)\right)}{u}\right)\right] \tilde{f}_{j}(\tau) . }
\end{aligned}
$$

Fazendo $u \rightarrow 0$ nas duas equações acima obtemos, respectivamente:

$$
a_{j} \tau_{j}+\lim _{u \rightarrow 0}\left[u^{-\alpha_{j}} R_{j}\left(X_{u}(\tau)\right)\right]=a_{1}^{\bar{\alpha}_{j}} \tilde{f}_{j}(\tau)
$$

e

$$
A_{j} \tau_{j}+\lim _{u \rightarrow 0}\left[u^{-\bar{\alpha}_{j}} B\left(-\bar{\beta}_{j} \ln u\right) R_{j}\left(X_{u}(\tau)\right)\right]=a_{1}^{\alpha_{j}} B\left(\bar{\beta}_{j} \ln a_{1}\right) \tilde{f}_{j}(\tau)
$$

Observe agora que, se mostrarmos que ambos os limites acima são nulos, então concluiremos a parte $\mathrm{A}$ do teorema, isto é, teremos que $\tilde{f}_{j}$ é linear. É suficiente mostrarmos que $\lim _{u \rightarrow 0} u^{-\bar{\alpha}_{j}} Q_{j}\left(X_{u}(\tau)\right)=\lim _{u \rightarrow 0} u^{-\bar{\alpha}_{j}} P_{j}\left(X_{u}(\tau)\right)=0$.

Mostremos primeiramente que $\lim _{u \rightarrow 0} u^{-\bar{\alpha}_{j}} Q_{j}\left(X_{u}(\tau)\right)=0$. De fato, temos que $\left\|u^{-\bar{\alpha}_{j}} Q_{j}\left(X_{u}(\tau)\right)\right\|=\left\|u^{l-\alpha_{j}}\right\|\left\|\frac{Q_{j}\left(X_{u}(\tau)\right)}{u^{l}}\right\|$. Como $l>\vec{\alpha}_{j}$ segue que

$$
\lim _{u \rightarrow 0}\left\|u^{-\bar{\alpha}_{j}} Q_{j}\left(X_{u}(\tau)\right)\right\|=0,
$$


como queríamos.

Mostremos agora que $\lim _{u \rightarrow 0} u^{-\alpha_{j}} P_{j}\left(X_{u}(\tau)\right)=0$. Como a notação é muito carregada em termos de índices, suporemos que $\mu_{p} \notin \mathbb{R}$ para algum $1<p \leq k$ e que $\mu_{j} \in \mathbb{R}$ para todo $j \neq p$, ou seja, que $X$ tenha apenas um autovalor complexo. Os demais casos, embora mais complicados devido aos índices, são análogos.

Seja $x=\left(x_{1}, \ldots, x_{p}, \ldots, x_{n-1}\right)$, onde $x_{p}=\left(x_{p 1}, x_{p 2}\right)$. Como $P(x)$ é um polinômio de grau maior ou igual a 2 e menor ou igual a $l$, escrevemos

$$
P(x)=\sum_{2 \leq|\sigma| \leq l} a_{\sigma} x^{\sigma},
$$

onde $\sigma=\left(\sigma_{1}, \ldots, \sigma_{p}, \ldots, \sigma_{n-1}\right)$ com $\sigma_{p}=\left(\sigma_{p 1}, \sigma_{p 2}\right), \quad \sigma_{j}, \sigma_{p 1}, \sigma_{p 2} \in \mathbf{Z}_{+}$ $(1 \leq j \leq n-1$ com $j \neq p)$ e $|\sigma|=\sigma_{1}+\ldots+\sigma_{p-1}+\sigma_{p 1}+\sigma_{p 2}+\sigma_{p+1}+\ldots+\sigma_{n-1}$.

$$
\begin{aligned}
& \text { Observe que } x^{\sigma}=x_{1}^{\sigma_{1}} x_{2}^{\sigma_{2}} \cdots x_{p 1}^{\sigma_{p 1}} x_{p 2}^{\sigma_{p 2}} \cdots x_{n-1}^{\sigma_{n-1}} \text { e } a_{\sigma}=\frac{\partial^{|\sigma|} f}{\partial x^{\sigma}}(x) . \text { Logo, } \\
& u^{-\bar{\alpha}_{j}} P_{j}\left(X_{u}(\tau)\right)=u^{-\bar{\alpha}_{j}} P_{j}\left(u, u^{\bar{\alpha}_{2}} \tau_{2}, \ldots, u^{\bar{\alpha}_{p}} B\left(\tilde{\beta}_{p} \ln u\right) \tau_{p}, \ldots, u^{\bar{\alpha}_{n-1}} \tau_{n-1}\right)= \\
& =u^{-\alpha_{j}} \sum_{2 \leq|\sigma| \leq l} a_{\sigma} u^{\sigma_{1}}\left(u^{\bar{\alpha}_{2}} \tau_{2}\right)^{\sigma_{2}} \cdots\left(u^{\bar{\alpha}_{p}} B\left(\bar{\beta}_{p} \ln u\right) \tau_{p}\right)^{\sigma_{p}} \cdots\left(u^{\bar{\alpha}_{n-1}} \tau_{n-1}^{\sigma_{n-1}}\right)= \\
& =u^{-\alpha_{j}} \sum_{2 \leq|\sigma| \leq l} a_{\sigma} u^{\sigma_{1}} u^{\alpha_{2} \sigma_{2}} \tau_{2}^{\sigma_{2}} \cdots\left\{u^{\bar{\alpha}_{p}}\left[\begin{array}{rr}
\cos \left(\bar{\beta}_{p} \ln u\right) & \operatorname{sen}\left(\bar{\beta}_{p} \ln u\right) \\
-\operatorname{sen}\left(\bar{\beta}_{p} \ln u\right) & \cos \left(\bar{\beta}_{p} \ln u\right)
\end{array}\right]\left[\begin{array}{c}
\tau_{p 1} \\
\tau_{p 2}
\end{array}\right]\right\}^{\sigma_{p}} \cdots \\
& \cdots u^{\bar{\alpha}_{n-1} \sigma_{n-1}} \tau_{n-1}^{\sigma_{n-1}}= \\
& =u^{-\bar{\alpha}_{j}} \sum_{2 \leq|\sigma| \leq l} a_{\sigma} u^{\sigma_{1}} u^{\bar{\alpha}_{2} \sigma_{2}} \tau_{2}^{\sigma_{2}} \cdots\left[u^{\bar{\alpha}_{p}}\left(\cos \left(\bar{\beta}_{p} \ln u\right) \tau_{p 1}+\operatorname{sen}\left(\bar{\beta}_{p} \ln u\right) \tau_{p 2}\right)\right]^{\sigma_{p 1}} \\
& \cdot\left[u^{\bar{\alpha}_{p}}\left(-\operatorname{sen}\left(\vec{\beta}_{p} \ln u\right) \tau_{p 1}+\cos \left(\bar{\beta}_{p} \ln u\right) \tau_{p 2}\right)\right]^{\sigma_{p 2}} \cdots u^{\bar{Q}_{n-1} \sigma_{n-1}} \tau_{n-1}^{\sigma_{n-1}}=
\end{aligned}
$$




$$
\begin{gathered}
=u^{-\alpha_{j}} \sum_{2 \leq|\sigma| \leq l} a_{\sigma} u^{\sigma_{1}+\alpha_{2} \sigma_{2}+\alpha_{3} \sigma_{3}+\cdots+\alpha_{p}\left(\sigma_{p 1}+\sigma_{p 2}\right)+\cdots+\alpha_{n-1} \sigma_{n-1}} \cdot \\
\cdot\left(\tau_{p 1} \cos \left(\bar{\beta}_{p} \ln u\right)+\tau_{p 2} \operatorname{sen}\left(\bar{\beta}_{p} \ln u\right)\right)^{\sigma_{p 1}}\left(-\tau_{p 1} \operatorname{sen}\left(\bar{\beta}_{p} \ln u\right)+\tau_{p 2} \cos \left(\bar{\beta}_{p} \ln u\right)\right)^{\sigma_{p 2}} \\
\cdot\left(\tau_{2}^{\sigma_{2}} \cdots \tau_{p-1}^{\sigma_{p-1}} \tau_{p+1}^{\sigma^{p+1}} \cdots \tau_{n-1}^{\sigma_{n-1}}\right)=\sum_{2 \leq|\sigma| \leq l} a_{\sigma} u^{\sigma_{1}+\alpha_{2} \sigma_{2}+\alpha_{3} \sigma_{3}+\cdots+\alpha_{p}\left(\sigma_{p 1}+\sigma_{p 2}\right)+\cdots \alpha_{n-1} \sigma_{n-1}-\alpha_{j}} \cdot \Delta,
\end{gathered}
$$

onde

$$
\begin{aligned}
\Delta=\left(\tau_{p 1} \cos \left(\vec{\beta}_{p} \ln u\right)+\tau_{p 2} \operatorname{sen}\left(\bar{\beta}_{p} \ln u\right)\right)^{\sigma_{p 1}} & \\
& \left(-\tau_{p 1} \operatorname{sen}\left(\bar{\beta}_{p} \ln u\right)+\tau_{p 2} \cos \left(\bar{\beta}_{p} \ln u\right)\right)^{\sigma_{p 2}} \tau_{2}^{\sigma_{2}} \cdots \tau_{p \rightarrow 1}^{\sigma_{p-1}} \tau_{p+1}^{\sigma_{p+1}} \cdots \tau_{n-1}^{\sigma_{n-1}}
\end{aligned}
$$

Pondo

$$
\vec{\alpha} \sigma=\sigma_{1}+\bar{\alpha}_{2} \sigma_{2}+\bar{\alpha}_{3} \sigma_{3}+\cdots+\bar{\alpha}_{p}\left(\sigma_{p 1}+\sigma_{p 2}\right)+\cdots \bar{\alpha}_{n-1} \sigma_{n-1}
$$

segue que

$$
u^{-\bar{\alpha}_{j}} P_{j}\left(X_{u}(\tau)\right)=\sum_{2 \leq|\sigma| \leq l} a_{\sigma} u^{\bar{\alpha} \sigma-\bar{\alpha}_{j}} \cdot \Delta .
$$

Como os autovalores $\left\{\alpha_{1}, \alpha_{2}, \ldots, \alpha_{p-1}, \alpha_{p}+i \beta_{p}, \alpha_{p}-i \alpha_{p}, \alpha_{p+1}, \ldots, \alpha_{n-1}\right\}$ satisfazem as condições de não ressonância, temos que

$$
\alpha_{j} \neq m_{1} \alpha_{1}+m_{2} \alpha_{2}+\cdots+m_{p}\left(\alpha_{p}+i \beta_{p}\right)+\bar{m}_{p}\left(\alpha_{p}-i \beta_{p}\right)+\cdots+m_{n-1} \alpha_{n-1}
$$

$\operatorname{com} m_{1}+m_{2}+\cdots+m_{p}+\bar{m}_{p}+\cdots+m_{n-1} \geq 2, \quad m_{j} \in \mathbb{Z}_{+}$.

Suponha que $\bar{\alpha} \sigma-\bar{\alpha}_{j}=0$. Então

$$
\sigma_{1} \alpha_{1}+\sigma_{2} \alpha_{2}+\sigma_{3} \alpha_{3}+\cdots+\left(\sigma_{p 1}+\sigma_{p 2}\right) \alpha_{p}+\cdots+\sigma_{n-1} \alpha_{n-1}=\alpha_{j}
$$


ou ainda,

$$
\begin{aligned}
& \sigma_{1} \alpha_{1}+\sigma_{2} \alpha_{2}+\sigma_{3} \alpha_{3}+\cdots+\left(\frac{\sigma_{p 1}+\sigma_{p 2}}{2}\right) \alpha_{p}+ \\
& +\left(\frac{\sigma_{p 1}+\sigma_{p 2}}{2}\right) i \beta_{p}+\left(\frac{\sigma_{p 1}+\sigma_{p 2}}{2}\right) \alpha_{p}-\left(\frac{\sigma_{p 1}+\sigma_{p 2}}{2}\right) i \beta_{p}+\cdots+\sigma_{n-1} \alpha_{n-1}=\alpha_{j}
\end{aligned}
$$

ou ainda,

$$
\begin{aligned}
& \sigma_{1} \alpha_{1}+\sigma_{2} \alpha_{2}+\sigma_{3} \alpha_{3}+\cdots+\left(\frac{\sigma_{p 1}+\sigma_{p 2}}{2}\right)\left(\alpha_{p}+i \beta_{p}\right)+ \\
& \quad+\left(\frac{\sigma_{p 1}+\sigma_{p 2}}{2}\right)\left(\alpha_{p}-i \beta_{p}\right)+\sigma_{n-1} \alpha_{n-1}=\alpha_{j}
\end{aligned}
$$

Absurdo pois

$$
\begin{aligned}
\sigma_{1}+\sigma_{2}+\sigma_{3}+\cdots+\left(\frac{\sigma_{p 1}+\sigma_{p 2}}{2}\right)+\left(\frac{\sigma_{p 1}+\sigma_{p 2}}{2}\right)+\cdots+\sigma_{n-1} & = \\
& =\sigma_{1}+\sigma_{2}+\sigma_{3}+\cdots+\sigma_{p 1}+\sigma_{p 2}+\cdots+\sigma_{n-1}=|\sigma| \geq 2
\end{aligned}
$$

Portanto $\bar{\alpha} \sigma-\bar{\alpha}_{j} \neq 0$. Consequentemente,

$\lim _{u \rightarrow 0} u^{-\bar{\alpha}_{j}} P_{j}\left(X_{u}(\tau)\right)=\lim _{u \rightarrow 0}\left(\sum_{2 \leq|\sigma| \leq l} a_{\sigma} u^{\bar{\alpha} \sigma-\bar{\alpha}_{j}} \Delta\right)=\sum_{2 \leq|\sigma| \leq l} \lim _{u \rightarrow 0}\left(a_{\sigma} u^{\bar{\alpha} \sigma-\bar{\alpha}_{j}} \Delta\right)=\left\{\begin{array}{l}0 \\ \infty .\end{array}\right.$

Observe que das equações 2.48 e 2.49 segue que o limite acima existe (é claro que, se $\vec{\alpha} \sigma-\bar{\alpha}_{j}<0$ então $a_{\sigma}=0$ ). Logo, $\lim _{u \rightarrow 0} u^{-\bar{\alpha}_{j}} P_{j}\left(X_{u}(\tau)\right)=0$.

Concluímos assim que $\tilde{f}_{j}$ é linear.

CASO B- Suponhamos que $\beta_{1} \neq 0$.

Conforme prova do Teorema 2.1 temos que $\tilde{f}_{\theta}$ é uma rotação. Mostremos que $\tilde{f}_{j}$ é linear, $j=2, \ldots, k$. Das equações 2.43 e 2.46 temos, respectivamente: 
- se $\mu_{j} \in \mathbb{R}$ :

$$
a_{j} \tau_{j}+r^{-\bar{\alpha}_{j}} R_{j}\left(X_{r}(\tau)\right)=\left[\delta+\frac{\bar{R}\left(X_{r}(\tau)\right)}{r}\right]^{\bar{\alpha}_{j}} \tilde{f}_{j}(\tau)
$$

- se $\mu_{j} \notin \mathbb{R}$ :

$$
\begin{aligned}
& A_{j} \tau_{j}+r^{-\bar{\alpha}_{j}} B\left(-\bar{\beta}_{j} \ln r\right) r_{j} R_{j}\left(X_{r}(\tau)\right)= \\
& =\left[\delta+\frac{\bar{R}\left(X_{r}(r)\right)}{r}\right]^{\bar{\alpha}_{j}} B\left[\bar{\beta}_{j} \ln \left(\delta+\frac{\bar{R}\left(X_{r}(\tau)\right)}{r}\right)\right] \tilde{f}_{j}(\tau) .
\end{aligned}
$$

Fazendo $r \rightarrow 0$ nas duas expressões acima e utilizando raciocínio análogo ao do caso $\mathrm{A}$ chegamos à conclusão de que $\tilde{f}_{j}$ é linear, o que conclui a prova do Teorema 2.2 . 


\section{Capítulo 3}

\section{Dinâmica da aplicação induzida}

\section{para $X \in \mathcal{H}\left(\mathbb{R}^{2}\right)$}

Neste último capítulo faremos um estudo detalhado da aplicação induzida por um difeomorfismo local $f: U \subset \mathbb{R}^{2} \rightarrow \mathbb{R}^{2}$ que preserva órbitas de $X \in \mathcal{H}\left(\mathbb{R}^{2}\right)$, ou seja, estudaremos a aplicação $\bar{f}$ que aparece no diagrama comutativo abaixo,

$$
\begin{array}{ccc}
U_{0} & \stackrel{f}{\longrightarrow} & \mathbb{R}_{0}^{2} \\
\downarrow^{p} & & \downarrow^{p} \\
\frac{U_{0}}{X} \stackrel{\bar{f}}{\longrightarrow} & \frac{\mathbb{R}_{0}^{2}}{X}
\end{array}
$$

onde $p$ é a aplicação quociente.

Estamos interessados em saber quando $\bar{f}$ é estruturalmente estável. Separaremos nosso estudo nos seguintes casos, dependentes dos autovalores do campo $X$ :

1. autovalores complexos conjugados;

2. autovalores reais:

(a) autovalores distintos e de mesmo sinal; 
(b) autovalores de sinais opostos e

(c) autovalores iguais.

Observe que quando os autovalores de $X$ forem como em 1 ou 2(a), a Proposição 2.1 do Capítulo 2 nos fornece informações sobre $\vec{f}$. Para os demais casos tal resultado não se aplica. Nos casos em que o campo $X$ tem índice 2 (ou 0 ), que são os casos $1,2(\mathrm{a})$ e $2(\mathrm{c}), \frac{\mathbb{R}_{0}^{2}}{X}$ pode ser 'realizado' como sendo a $S^{1}$ (isto fica bastante claro com a observação do Lema 1.1 do Capítulo 1). Para estes casos temos o Teorema 1.6 do Capítulo 1 , devido a Peixoto, que dá ưna caracterização dos difeomorfismos definidos sobre $S^{1}$ estruturalmente estáveis. Estudemos então cada caso separadamente.

\subsection{Autovalores complexos conjugados}

Da Proposição 2.1 do Capítulo 2 temos que $\bar{f}: S^{1} \rightarrow S^{1}$ é uma rotação e consequentemente, conforme Observação 2.4 (cap.2), $\Omega(\bar{f})=S^{1}$. Segue então do Teorema 1.6 que $\bar{f}$ não é estruturalmente estável.

\subsection{Autovalores reais}

Seja $f \in \operatorname{Difr}(U, X), r \geq 1$. Quando os autovalores $\alpha_{1}$ e $\alpha_{2}$ do campo $X$ são reais, então as órbitas do campo $X$ são conforme Figura 3.1, onde (i) corresponde ao caso em que os autovalores são distintos e de mesmo sinal, (ii) ao caso em que os autovalores são de sinais opostos e tanto (iii) quanto (iv) para o caso em que os autovalores são iguais.

Supondo $X$ na forma de Jordan, em (i), (ii) e (iii) temos que $X=\left(\begin{array}{cc}\alpha_{1} & 0 \\ 0 & \alpha_{2}\end{array}\right)$, sendo que em (iii) $\alpha_{1}=\alpha_{2}=\alpha$. Em (iv) temos $X=\left(\begin{array}{cc}\alpha & 1 \\ 0 & \alpha\end{array}\right)$. 


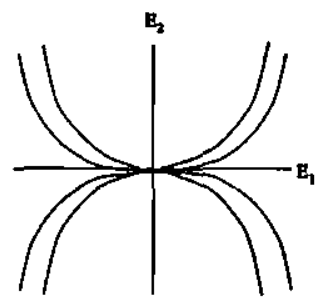

(i)

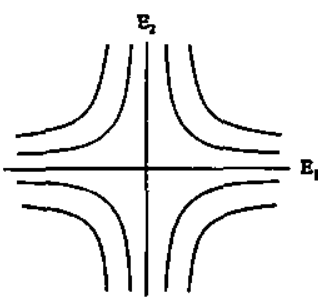

(ii)

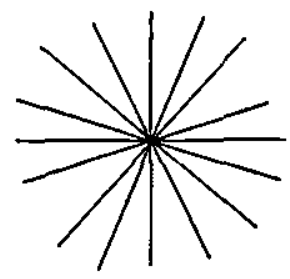

(iii)

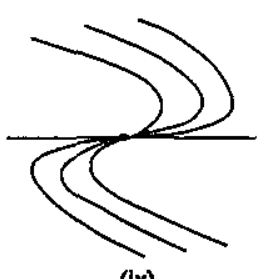

(iv)

Figura 3.1: Órbitas do campo X cujos autovalores $\alpha_{1}$ e $\alpha_{2}$ são reais satisfazendo: (i) $\alpha_{2}<\alpha_{1}<0$ (ou $0<\alpha_{1}<\alpha_{2}$ ); (ii) $\alpha_{1}<0<\alpha_{2}$; (iii) e (iv) $\alpha_{1}=\alpha_{2}=\alpha$. O subespaço $E_{j}$ é o autoespaço associado ao autovalor $\alpha_{j}, j=1,2$.

Em (i) sabemos do Lema 2.2 do Capítulo 2, que $E_{2}$ é invariante por $f$. Assim, $y^{ \pm}=(0, \pm 1)$ são pontos periódicos de $\vec{f}$ de período 1 ou 2 . Para o caso em que os autovalores são distintos e de sinais opostos, como em (ii), o seguinte lema nos diz que tanto $E_{1}$ quanto $E_{2}$ são invariantes por $f$ :

Lema 3.1 Sejam $f \in \operatorname{Dif}^{r}(U, X), r \geq 1, U$ aberto de $\mathbb{R}^{2}$ contendo a origem, onde $X \in \mathcal{H}^{r}\left(\mathbb{R}^{2}\right)$ é tal que $X=\left(\begin{array}{cc}\alpha_{1} & 0 \\ 0 & \alpha_{2}\end{array}\right)$, com $\alpha_{1}<0<\alpha_{2}$. Se $E_{j}$ é o autoespaço associado ao autovalor $\alpha_{j}$ então $f\left(U \cap E_{j}\right) \subset E_{j}$, para $j=1,2$.

\section{Demonstração:}

Seja $p \in U \cap E_{1}$. Mostremos que $f(p) \in E_{1}$. Segue da Proposição 1.2 do Capítulo 1 que

$$
\lim _{t \rightarrow \infty} X_{t}(p)=0
$$

Como $f$ é contínua,

$$
\lim _{t \rightarrow \infty} f\left(X_{t}(p)\right)=f(0)=0 .
$$

Como $f$ preserva órbitas de $X$, ou seja, $f\left(X_{t}(p)\right)=X_{\sigma(t)}(f(p))$, segue que

$$
\lim _{t \rightarrow \infty} X_{\sigma(t)}(f(p))=0
$$


Observando que $\sigma$ é um homeomorfismo crescente (e que $f$ preserva a orientação das órbitas) concluímos que $f(p) \in E_{1}$. A prova para a invariância de $E_{2}$ é análoga.l

Observaçāo 3.1 Se $X$ for como em (iii), supondo que a aplicação induzida $\vec{f}$ : $S^{1} \rightarrow S^{1}$ tenha um ponto fixo, digamos $y^{+}$, então a órbita de $X$ por este ponto é invariante por $f$, assim como também a reta $L=\left\{\lambda y^{+}, \lambda \in \mathbb{R}\right\}$. Logo, tomando $\{u, v\}$ como base para $\mathbb{R}^{2}$, sendo $v \in L$ e $u$ qualquer vetor linearmente independente com $v$, temos que as coordenadas de $y^{+} \in S^{1}$ com relação a esta base são $(0,1)$. Temos ainda que $y^{-}=(0,-1)$ também é um ponto fixo de $\bar{f}$.

Como estamos interessados em saber se $\bar{f}: \frac{U_{0}}{X} \rightarrow \frac{\mathbb{R}_{0}^{n}}{X}$ é estruturalmente estável, devemos verificar se os pontos periódicos de $\bar{f}$ são hiperbólicos. Para tanto precisaremos encontrar a derivada de $\vec{f}$ em seus pontos periódicos. Para os casos em que as órbitas do campo $X$ são como uma das figuras (i), (ii) ou (iii) anteriores temos o seguinte resultado, que é um caso particular do Lema 3.2 de [2].

Lema 3.2 Seja $f \in \operatorname{Dif}^{r}(U, X), r \geq 1$, onde $X$ é um campo linear hiperbólico em $\mathbb{R}^{2}$, com autovalores $\alpha_{1}$ e $\alpha_{2}$ reais e cuja matriz é diagonalizável. Supondo que $x^{ \pm}=( \pm 1,0)$ e $y^{ \pm}=(0, \pm 1)$ são pontos fixos de $\bar{f}$, então $\bar{f}^{\prime}\left(x^{ \pm}\right)=a^{-\frac{\alpha_{2}}{\alpha_{1}}}$ b e $\bar{f}^{\prime}\left(y^{ \pm}\right)=a b^{-\frac{\alpha_{1}}{\alpha_{2}}}$, onde $a=\frac{\partial f_{1}}{\partial x_{1}}(0)>0$ eb $=\frac{\partial f_{2}}{\partial x_{2}}(0)>0$.

\section{Demonstraçāo:}

Consideremos primeiramente o caso em que $\alpha_{1} \neq \alpha_{2}$.

Podemos escrever $f(x)=D f(0)(x)+R(x)$, onde $D f(0)=\left(\begin{array}{ll}a & c \\ d & b\end{array}\right)$ e $\lim _{x \rightarrow 0} \frac{R(x)}{|x|}=0$, $\operatorname{com} x=\left(x_{1}, x_{2}\right)$. Segue dos Lemas 2.1 e 3.1 que

$$
f\left(x_{1}, x_{2}\right)=\left(f_{1}\left(x_{1}, x_{2}\right), f_{2}\left(x_{1}, x_{2}\right)\right)=\left(a x_{1}+R_{1}\left(x_{1}, x_{2}\right), b x_{2}+R_{2}\left(x_{1}, x_{2}\right)\right)
$$


(de fato, se $\alpha_{1}$ e $\alpha_{2}$ tiverem o mesmo sinal, do Lema 2.1 (iii) segue que $D f(0)$ comuta com $X$ e daí $c=d=0$; se $\alpha_{1}$ e $\alpha_{2}$ forem de sinais distintos então, das invariâncias de $E_{1}$ e $E_{2}$ por $f$, dadas no Lema 3.1, segue que $0=f_{2}\left(x_{1}, 0\right)=d x_{1}+R_{2}\left(x_{1}, 0\right)$ e assim $d=0$ e que $0=f_{1}\left(0, x_{2}\right)=c x_{2}+R_{1}\left(0, x_{2}\right)$, ou seja, $\left.c=0\right)$.

Como $b>0$ segue que $H_{+}=\left\{\left(x_{1}, x_{2}\right) \in \mathbb{R}^{2} ; \quad x_{2}>0\right\}$ é invariante por $f$. De fato, seja $\left(x_{1}, x_{2}\right) \in H_{+}$. Como $f\left(x_{1}, x_{2}\right)=\left(f_{1}\left(x_{1}, x_{2}\right), f_{2}\left(x_{1}, x_{2}\right)\right)$, devemos mostrar que $f_{2}\left(x_{1}, x_{2}\right)>0$. Mas isto é imediato visto que $f_{2}\left(x_{1}, x_{2}\right)=b x_{2}+R_{2}\left(x_{1}, x_{2}\right), b>0$ e $R_{2}\left(x_{1}, x_{2}\right)$ tem termos de ordem $>1$, com $\left(x_{1}, x_{2}\right)$ em uma vizinhança da origem.

Calculemos $\vec{f}^{\prime}\left(y^{+}\right)$. Fixemos um ponto $(0, k)$, com $0<k<1$ e seja $T_{k}=\left\{\left(x_{1}, x_{2}\right) \in \mathbb{R}^{2} ; \quad x_{2}=k\right\}$. Defina $\psi_{k}:\left\{\left(x_{1}, x_{2}\right) \in \mathbb{R}^{2} ; x_{2}>0\right\} \longrightarrow \mathbb{R}$ por

$$
\psi_{k}\left(X_{t}\left(x_{1}, x_{2}\right)\right)=\pi_{1}\left(X_{t}\left(x_{1}, x_{2}\right) \cap T_{k}\right)
$$

Então $\psi_{k}\left(x_{1}, x_{2}\right)=x_{1}\left(\frac{k}{x_{2}}\right)^{\frac{\alpha_{1}}{\alpha_{2}}}$. De fato, como $X_{t}\left(x_{1}, x_{2}\right)=\left(e^{\alpha_{1} t} x_{1}, e^{\alpha_{2} t} x_{2}\right)$ temos que $e^{\alpha_{2} t} x_{2}=k \Rightarrow t=\frac{1}{\alpha_{2}} \ln \left(\frac{k}{x_{2}}\right)$ e então, $e^{\alpha_{1} t} x_{1}=x_{1}\left(\frac{k}{x_{2}}\right)^{\frac{\alpha_{1}}{\alpha_{2}}}$.

Temos que $\psi_{k}$ induz a seguinte carta de $\frac{U_{0}}{X}$ em uma vizinhança de $y^{+}$:

$$
\begin{gathered}
\mathbf{x}: \frac{U_{0}}{X} \longrightarrow \mathbb{R} \\
\mathbf{x}\left(x_{1}, x_{2}\right)=\psi_{k}\left(x_{1}, x_{2}\right) .
\end{gathered}
$$

Assim, sabendo que $H_{+}$é invariante por $f$, temos que $\bar{f}$ nesta carta é dada por:

$$
\begin{aligned}
\tilde{f}(\tau)=\psi_{k}(f(\tau, k)) & =\psi_{k}\left(a \tau+R_{1}(\tau, k), b k+R_{2}(\tau, k)\right)= \\
& =\left[a \tau+R_{1}(\tau, k)\right]\left[\frac{k}{b+R_{2}(\tau, k)}\right]^{\frac{\alpha_{1}}{\alpha_{2}}}=\left(f_{1}(\tau, k)\right)\left(\frac{k}{f_{2}(\tau, k)}\right)^{\frac{\alpha_{1}}{\alpha_{2}}} .
\end{aligned}
$$


Dividindo ambos os membros por $\tau$, temos

$$
\frac{\tilde{f}(\tau)}{\tau}=\frac{f_{1}(\tau, k)}{\tau}\left(\frac{k}{f_{2}(\tau, k)}\right)^{\frac{\alpha_{1}}{\alpha_{2}}} .
$$

Fazendo $\tau \rightarrow 0$ nesta última expressão, obtemos

$$
\bar{f}^{\prime}\left(y^{+}\right)=\frac{\partial f_{1}}{\partial x_{1}}(0, k)\left(\frac{k}{f_{2}(0, k)}\right)^{\frac{\alpha_{1}}{\alpha_{2}}}
$$

visto que

$$
\begin{aligned}
& \lim _{\tau \rightarrow 0} \frac{f_{1}(\tau, k)}{\tau}=\lim _{\tau \rightarrow 0} \frac{f_{1}(\tau, k)-f_{1}(0, k)}{\tau}= \\
&=\lim _{\tau \rightarrow 0} \frac{f_{1}((0, k)+\tau(1,0))-f_{1}(0, k)}{\tau}=\frac{\partial f_{1}}{\partial x_{1}}(0, k) .
\end{aligned}
$$

Mostremos que $\bar{f}^{\prime}\left(y^{+}\right)$não depende da carta escolhida. Fixando $k_{0} \neq k, k_{0}>0$, tomemos uma outra carta

$$
\mathbf{x}_{0}: \frac{U_{0}}{X} \longrightarrow \mathbb{R}
$$

onde $\mathbf{x}_{0}\left(x_{1}, x_{2}\right)=\psi_{k_{0}}\left(x_{1}, x_{2}\right)$. Seja $\tilde{f}_{0}=\mathbf{x}_{0} \bar{f} \mathbf{x}_{0}^{-1}$. Devemos mostrar que $\tilde{f}_{0}^{\prime}(0)=$ $\tilde{f}^{\prime}(0)$, onde $\tilde{f}=\mathbf{x} \bar{f} \mathbf{x}^{-1}$.

Temos que

$$
\begin{aligned}
\tilde{f}_{0}=\mathbf{x}_{0}\left(\mathbf{x}^{-1} \mathbf{x}\right) \bar{f}\left(\mathbf{x}^{-1} \mathbf{x}\right) \mathbf{x}_{0}^{-1}=\left(\mathbf{x}_{0} \mathbf{x}^{-1}\right) \mathbf{x} \bar{f} \mathbf{x}^{-1}\left(\mathbf{x x}_{0}^{-1}\right)= \\
=\left(\mathbf{x x}_{0}^{-1}\right)^{-1} \mathbf{x} \bar{f} \mathbf{x}^{-1}\left(\mathbf{x x}_{0}^{-1}\right)=\left(\mathbf{x x}_{0}^{-1}\right)^{-1} \tilde{f}\left(\mathbf{x x}_{0}^{-1}\right) .
\end{aligned}
$$

Assim,

$$
\begin{aligned}
& \tilde{f}_{0}^{\prime}(0)=\frac{1}{\left(\mathbf{x x}_{0}^{-1}\right)^{\prime}\left(\tilde{f}\left(\mathrm{xx}_{0}^{-1}\right)(0)\right)} \cdot \tilde{f}^{\prime}\left(\mathrm{xx}_{0}^{-1}\right)(0) \cdot\left(\mathrm{xx}_{0}^{-1}\right)^{\prime}(0)= \\
& \quad=\frac{1}{\left(\mathrm{xx}_{0}^{-1}\right)^{\prime}(0)} \cdot \tilde{f}^{\prime}(0) \cdot\left(\mathrm{xx}_{0}^{-1}\right)^{\prime}(0)=\tilde{f}^{\prime}(0)
\end{aligned}
$$

como queríamos. 
Então, como $\bar{f}\left(y^{+}\right)$não depende de $k$, fazendo $k \rightarrow 0$ na equação 3.2 , obtem̧os:

$$
\bar{f}^{\prime}\left(y^{+}\right)=a \lim _{k \rightarrow 0}\left(b+\frac{R_{2}(0, k)}{k}\right)^{-\frac{\alpha_{1}}{\alpha_{2}}} .
$$

Portanto,

$$
\bar{f}^{\prime}\left(y^{+}\right)=a b^{-\frac{\alpha_{1}}{\alpha_{2}}} \text {. }
$$

Um raciocínio análogo nos dá que

$$
\bar{f}^{\prime}\left(y^{-}\right)=a b^{-\frac{\alpha_{1}}{\alpha_{2}}} \text {. }
$$

Para calcularmos $\bar{f}^{\prime}\left(x^{ \pm}\right)$, procedemos de maneira análoga, tomando $T_{k}=\left\{\left(x_{1}, x_{2}\right) \in\right.$ $\left.\mathbb{R}^{2} ; \quad x_{1}=k\right\}$ e definindo $\psi_{k}\left(x_{1}, x_{2}\right)=\pi_{2}\left(X_{t}\left(x_{1}, x_{2}\right) \cap T_{k}\right)$. Como $a>0$ temos que $H^{+}=\left\{\left(x_{1}, x_{2}\right) \in \mathbb{R}^{2} ; x_{1}>0\right\}$ é invariante por $f$. Com isso, obtemos

$$
\bar{f}^{\prime}\left(x^{ \pm}\right)=a^{-\frac{\alpha_{2}}{\alpha_{1}} b} \text {. }
$$

Suponhamos agora que $\alpha_{1}=\alpha_{2}=\alpha$.

Com relação à base $\{u, v\}$ dada na observação 3.1, podemos escrever

$$
f\left(x_{1}, x_{2}\right)=\left(a x_{1}+R_{1}\left(x_{1}, x_{2}\right), d x_{1}+b x_{2}+R_{2}\left(x_{1}, x_{2}\right)\right),
$$

visto que a reta $L$ é invariante por $f$. Com um raciocínio análogo ao anterior segue que

$$
\bar{f}^{\prime}\left(y^{ \pm}\right)=a b^{-\frac{\alpha_{1}}{\alpha_{2}}}=a b^{-1} \text {. }
$$

Analogamente,

$$
\bar{f}^{\prime}\left(x^{ \pm}\right)=a^{-\frac{\alpha_{2}}{\alpha_{1}} b=a^{-1} b},
$$

o que conclui a prova do lema.

Observaçāo 3.2 Como $\left|a b^{-\frac{\alpha_{1}}{\alpha_{2}}}\right|=1 \Leftrightarrow\left|a^{-\frac{\alpha_{2}}{\alpha_{1}}} b\right|=1$, temos que os pontos fixos $x^{ \pm} \mathrm{e}$ $y^{ \pm}$são hiperbólicos de $\bar{f} \Leftrightarrow\left|a b^{-\frac{\alpha_{1}}{\alpha_{2}}}\right| \neq 1 \Leftrightarrow \alpha_{2} \ln |a| \neq \alpha_{1} \ln |b|$.

Estamos em condições agora de completar nossa análise sobre a estabilidade estrutural de $\bar{f}$. 


\subsubsection{Autovalores distintos e de mesmo sinal}

Temos que $f\left(x_{1}, x_{2}\right)=\left(a x_{1}+R_{1}\left(x_{1}, x_{2}\right), b x_{2}+R_{2}\left(x_{1}, x_{2}\right)\right)$, onde $a=\frac{\partial f_{1}}{\partial x_{1}}(0)$ e $b=\frac{\partial f_{2}}{\partial x_{2}}(0)$. Vamos estudar os casos em que $a>0, a<0, b>0$ e $b<0$.

Como $E_{2}$ é invariante por $f$ então $y^{ \pm}$são pontos fixos de $\vec{f}$ ou de $\vec{f}^{2}$ conforme $b>0$ ou $b<0$, respectivamente. $\underline{\mathrm{Se} b}>0$, da Observação 3.2 segue que os pontos fixos $y^{ \pm}$são hiperbólicos de $\bar{f} \Leftrightarrow \alpha_{2} \ln |a| \neq \alpha_{1} \ln |b|$.

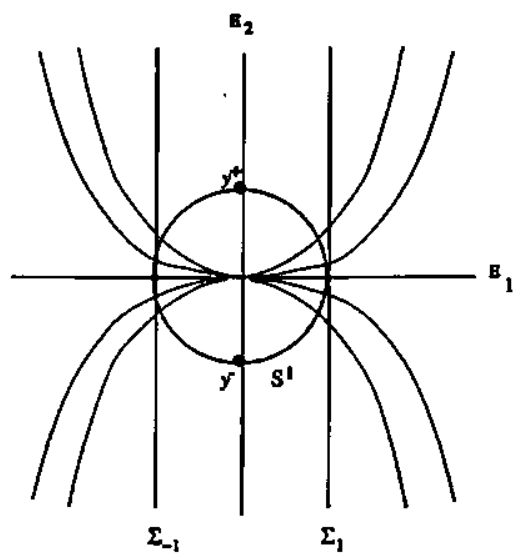

Figura 3.2: As órbitas de $X$ para o caso em que seus autovalores satisfazem $\alpha_{2}<\alpha_{1}<0$ e os espaços $\frac{\mathbb{R}_{0}^{2}}{X} \simeq S^{1}$ e $\Sigma=\Sigma_{1} \cup \Sigma_{-1}$.

$\underline{\text { Se } b<0}$ então $y^{ \pm}$são pontos fixos de $\bar{f}^{2}$. Pondo $f^{2}=g$, seja $A=\frac{\partial g_{1}}{\partial x_{1}}(0) \mathrm{e}$ $B=\frac{\partial g_{2}}{\partial x_{2}}(0)$. Um simples cálculo nos dá que $A=a^{2}$ e $B=b^{2}$. Assim, como $B>0$ segue do Lema 3.2 que $\left(\bar{f}^{2}\right)^{\prime}\left(y^{ \pm}\right)=A B^{-\frac{\alpha_{1}}{\alpha_{2}}}$, ou seja

$$
\left(\bar{f}^{2}\right)^{\prime}\left(y^{ \pm}\right)=a^{2}\left(b^{2}\right)^{-\frac{\alpha_{1}}{\alpha_{2}}} \text {. }
$$

Portanto $y^{ \pm}$são hiperbólicos de $\bar{f} \Leftrightarrow \alpha_{2} \ln |a| \neq \alpha_{1} \ln |b|$.

Supondo $\underline{a>0}$, da Proposição 2.1 do Capítulo 2, temos que $\tilde{f}(\tau)=a^{-\frac{\alpha_{2}}{\alpha_{1}}} b \tau+$ $c$, para $\tau \in \mathbb{R}$. Se $a^{-\frac{\alpha_{2}}{\alpha_{1}} b}=1$, então $y^{ \pm}$não são hiperbólicos e portanto $\bar{f}$ 
não é estruturalmente estável. Se $a^{-\frac{\alpha_{2}}{\alpha_{1}}} b \neq 1$, então $a^{-\frac{\alpha_{2}}{\alpha_{1}}} b \tau+c$ é afim, de onde concluímos que $\bar{f}$ tem mais dois pontos periódicos além de $y^{ \pm}$os quais denotaremos por $x^{ \pm}$. Temos que $x^{ \pm}$e $y^{ \pm}$serão hiperbólicos $\Leftrightarrow \mid a^{-\frac{\alpha_{2}}{\alpha_{1}} b \mid} \neq 1$, ou seja, $\alpha_{2} \ln |a| \neq \alpha_{1} \ln |b|$. Além disso, estes são os únicos pontos pertencentes ao conjunto $\Omega(\bar{f})$ pois $\tilde{f}$ é uma contração ou uma expansão na vizinhança de seus pontos periódicos.

Supondo $\underline{a<0}$, então $x^{ \pm}$são pontos fixos de $\bar{f}^{2}$, a qual está nas mesmas condições que $\bar{f}$ quando $a>0$ e assim, temos o mesmo raciocínio anterior.

Portanto, se os autovalores de $X$ são reais distintos e de mesmo sinal, do Teorema 1.6 do Capítulo 1 , segue que $\bar{f}$ é estruturalmente estável $\Leftrightarrow \alpha_{2} \ln |a| \neq \alpha_{1} \ln |b|$.

\subsubsection{Autovalores de sinais opostos}

Neste parágrafo, tomamos como referência o artigo [2].

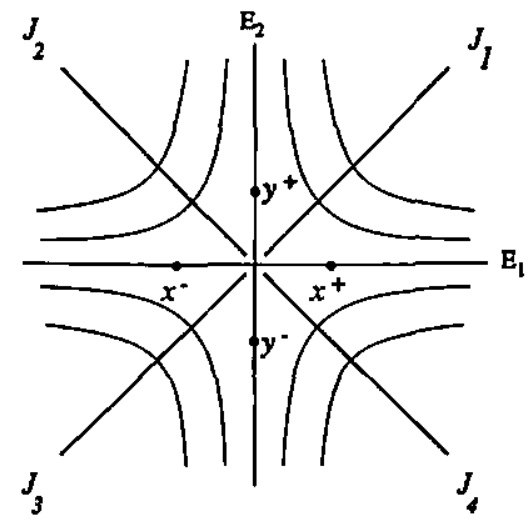

Figura 3.3: As órbitas de $X$ para o caso em que seus autovalores $\alpha_{1}$ e $\alpha_{2}$ são de sinais opostos e o espaço $\frac{\mathbb{R}_{0}^{2}}{X}$.

Neste caso o espaço $\frac{U_{0}}{X}$ pode ser "realizado" como a união de quatro intervalos: $J^{1}=\left\{\left(x_{1}, x_{2}\right) ; \quad x_{1}=x_{2}, x_{1}>0\right\} \cap U_{0} ; J^{2}=\left\{\left(x_{1}, x_{2}\right) ; \quad x_{1}=-x_{2}, x_{1}<0\right\} \cap U_{0} ;$ $J^{3}=\left\{\left(x_{1}, x_{2}\right) ; \quad x_{1}=x_{2}, x_{1}<0\right\} \cap U_{0}$ e $J^{4}=\left\{\left(x_{1}, x_{2}\right) ; \quad x_{1}=-x_{2}, x_{1}>0\right\} \cap U_{0}$ 
e quatro pontos $\left\{x^{+}, x^{-}, y^{+}, y^{-}\right\}$. Podemos tomar, sem perda de generalidade, $x^{ \pm}=( \pm 1,0)$ e $y^{ \pm}=(0, \pm 1)$. Note que cada um desses pontos corresponde às órbitas que são invariantes por $f$ ou $f^{2}$ dadas no Lema 3.1. Portanto os quatro pontos $\left\{x^{+}, x^{-}, y^{+}, y^{-}\right\}$são pontos fixos de $\bar{f}$ ou de $\bar{f}^{2}$. Note que $\frac{U_{0}}{X}$ é uma variedade não Hausdorff.

Sabendo que $f\left(x_{1}, x_{2}\right)=\left(a x_{1}+R_{1}\left(x_{1}, x_{2}\right) ; b x_{2}+R_{2}\left(x_{1}, x_{2}\right)\right)$, temos que:

- se $a>0$ então $x^{ \pm}$são pontos fixos de $\vec{f}$;

- se $a<0$ então $x^{ \pm}$são pontos periódicos de $\bar{f}$ de perfodo 2;

- se $b>0$ então $y^{ \pm}$são pontos fixos de $\bar{f}$;

- se $b<0$ então $y^{ \pm}$são pontos periódicos de $\bar{f}$ de período 2 .

$\mathrm{Da}$ Observação 3.2 do Lema 3.2 conclú́mos que os pontos $\left\{x^{ \pm}, y^{ \pm}\right\}$são hiperbólicos de $\bar{f} \Leftrightarrow \alpha_{2} \ln |a| \neq \alpha_{1} \ln |b|$. Note que estes são os únicos pontos pertencentes ao conjunto $\Omega(\bar{f})$ visto que $\bar{f}$ é uma contração ou uma expansão na vizinhança destes pontos.

Observe que o Teorema 1.6 do Capítulo 1 sobre estabilidade estrutural refere-se a difeomorfismos definidos em $S^{1}$. No caso em que os autovalores de $X$ são reais e de sinais opostos, embora $\bar{f}$ esteja definida em uma variedade não-compacta, sob a hipótese de que $\alpha_{2} \ln |a| \neq \alpha_{1} \ln |b|$, seus pontos periódicos são hiperbólicos e isto permite garantir a estabilidade estrutural de $\bar{f}$.

Observação 3.3 Em nossa definição de difeomorfismo que preserva órbitas de $X$ impomos que, além de $f$ mandar órbitas em órbitas, que $f$ também preserve a orientação das órbitas. Isto é necessário para mostrar que $E_{1}$ e $E_{2}$ são invariantes por $f$. Porém, desta maneira exclúmos alguns difeomorfismos, como por exemplo o seguinte: tomemos o campo $X(x, y)=(-x, y)$ e $f: \mathbb{R}^{2} \rightarrow \mathbb{R}^{2}$ dada por 
$f(x, y)=(-\lambda y, \lambda x), \quad 0<\lambda<1$. Então $\alpha_{1}=-1$ e $\alpha_{2}=1$ e $f$ preserva órbitas de $X$. Mas $f$ não preserva orientação das órbitas. Veja que $f\left(X_{t}(1,0)\right)=X_{-t}(0,1)$, ou seja, $f$ inverteu a orientação de $X_{t}(0,1)$. Neste exemplo os pontos $x^{+}, x^{-}, y^{+}$e $y^{-}$ são periódicos hiperbólicos de período 4 .

\subsubsection{Autovalores iguais}

Seja $\alpha$ o autovalor de $X$. O autoespaço $E$ gerado por $\alpha$ pode ter dimensão 1 ou 2 , casos em que as órbitas de $X$ são como as da Figura 3.1 (iii) e (iv), respectivamente. Temos o seguinte resultado que foi enunciado em [1], pg 90:

Proposição 3.1 Sejam $X$ um campo de vetores linear em $\mathbb{R}^{2}$ com autovalores reais, iguais e não nulos, $E$ o autoespaço gerado pelo autovalor e $f: U \rightarrow \mathbb{R}^{2}$ um difeomorfismo que preserva órbitas de $X$, sendo $U$ aberto de $\mathbb{R}^{2}$ contendo a origem. Então:

A: $S e \operatorname{dim} E=2$ e $f \in C^{1}$, temos dois casos:

i) Existe $z \neq 0$ tal que $f(\mathcal{O}(z))=\mathcal{O}(z)$. Neste caso, tomamos $L=\{\lambda z ; \lambda \in$ $\mathbb{R}\}, v \notin L, \Sigma_{v}=L+v$ e $\Sigma=\Sigma_{v} \cup \Sigma_{-v}$. Então, ou $\tilde{f}: \Sigma \rightarrow \Sigma$ ou $\tilde{f}^{2}: \Sigma \rightarrow \Sigma$ é afim;

ii) Para qualquer $z \neq 0, f(\mathcal{O}(z)) \cap \mathcal{O}(z)=\emptyset$. Neste caso, tomamos $\Sigma=S^{1}$. Então $\tilde{f}: S^{1} \rightarrow S^{1}$ é uma rotação.

B: Se $\operatorname{dim} E=1$ e $f \in C^{2}$ então tomando $\Sigma= \pm 1 \times E$ temos que ou $\tilde{f}: \Sigma \rightarrow \Sigma$ ou $\tilde{f}^{2}: \Sigma \rightarrow \Sigma$ é afim.

\section{Demonstraçāo:}

CASO A: Supondo que $\operatorname{dim} E=2$, as órbitas de $X$ são como as da Figura 3.1 (iii).

i) Seja $z \neq 0$ tal que $f(\mathcal{O}(z))=\mathcal{O}(z)$. Considere então a reta $L=\{\lambda z ; \quad \lambda \in \mathbb{R}\}$ e faça $\Sigma=\Sigma_{v} \cup \Sigma_{-v}$, onde $v \neq 0$ é tal que $v \notin L, \Sigma_{v}=L+v$ e $\Sigma_{-v}=L-v$. 


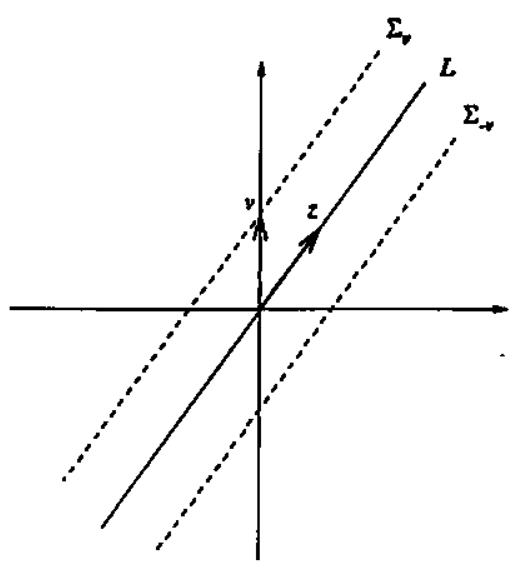

Figura 3.4: Como $f(\mathcal{O}(z))=\mathcal{O}(z)$ então $L$ é invariante por $f$.

Observe que $L$ é invariante por $f$. Seja $\{v, z\}$ base para $\mathbb{R}^{2}$. Dado $x \in \mathbb{R}^{2}$, sejam $\left(x_{1}, x_{2}\right)$ as coordenadas de $x$ com relação à base considerada. Como $f \in C^{1}$ temos $f(x)=D f(0) .(x)+R(x)$. Logo,

$$
f_{1}(x)=a x_{1}+c x_{2}+R_{1}(x) \text { e } f_{2}(x)=d x_{1}+b x_{2}+R_{2}(x),
$$

onde $a=\frac{\partial f_{1}}{\partial x_{1}} ; c=\frac{\partial f_{1}}{\partial x_{2}} ; d=\frac{\partial f_{2}}{\partial x_{1}}$ e $b=\frac{\partial f_{2}}{\partial x_{2}}$, todos avaliados em $(0,0)$, e $\lim _{x \rightarrow 0} \frac{R_{j}(x)}{\|x\|}=0, j=1,2$.

Como $L$ é invariante por $f$, concluímos que $c=0$. De fato, tomando $x=\left(0, x_{2}\right) \in L$ temos que $f(x)=\left(f_{1}(x), f_{2}(x)\right)=\left(0, f_{2}(x)\right)$. Daí $c x_{2}+R_{1}\left(0, x_{2}\right)=0$, de onde segue que $c=0$. Assim, temos que

$$
f_{1}(x)=a x_{1}+R_{1}(x) \quad \text { e } f_{2}(x)=d x_{1}+b x_{2}+R_{2}(x) .
$$

Deixaremos de justificar algumas passagens visto que o raciocínio para tais é análogo ao que já foi feito. Vamos supor que $a>0$. Então $\tilde{f}\left(\Sigma_{v}\right) \subset \Sigma_{v}$. Dado $(1, \tau) \in \Sigma_{v}$, por abuso de notação, denotaremos às vezes $(1, \tau)$ simplesmente por $\tau$.

A órbita de $X$ pelo ponto $(1, \tau)$ é dada por $X_{t}(\tau)=e^{\alpha t}(1, \tau)$. Fazendo $e^{\alpha t}=u$ obtemos $t=\frac{1}{\alpha} \ln u$. Daí $e^{\alpha \dot{t}} \tau=u \tau$. Agora temos uma expressão para a b́rbita do 
campo $X$ em função da primeira coordenada:

$$
X_{u}(\tau)=(u, u r)
$$

De 3.3 temos

$$
f\left(X_{u}(x)\right)=\left(a u+R_{1}\left(X_{u}(x)\right), d u+b u r+R_{2}\left(X_{u}(x)\right)\right)
$$

Por outro lado, como $f$ manda a órbita de $X$ pelo ponto $\tau$ na órbita pelo ponto $\tilde{f}(\tau)$, de 3.4 temos

$$
f\left(X_{u}(x)\right)=\left(a u+R_{1}\left(X_{u}(x)\right),\left[a u+R_{1}\left(X_{u}(x)\right)\right] \tilde{f}(\tau)\right) .
$$

Comparando as duas últimas expressões segue que

$$
d u+b u r+R_{2}\left(X_{u}(x)\right)=\left[a u+R_{1}\left(X_{u}(x)\right)\right] \tilde{f}(\tau) .
$$

Multiplicando por $u^{-1}$, obtemos

$$
d+b \tau+u^{-1} R_{2}\left(X_{u}(x)\right)=\left[a+u^{-1} R_{1}\left(X_{u}(x)\right)\right] \tilde{f}(\tau) .
$$

Derivando com relação a $r$

$$
b+D_{2} R_{2}\left(X_{u}(x)\right)=D_{2} R_{1}\left(X_{u}(x)\right) \tilde{f}(\tau)+\left[a+u^{-1} R_{1}\left(X_{u}(x)\right)\right] \tilde{f}^{\prime}(\tau) .
$$

Fazendo $u \rightarrow 0$ nesta última expressão concluímos que

$$
\tilde{f}(\tau)=\frac{b}{a} \tau+c
$$

onde $c$ é constante. Ou seja, $\tilde{f}$ é afim.

Se $a<0$ então $\tilde{f}^{2}$ está nas mesmas condições de $\tilde{f}$ quando $a>0$, de onde segue que

$$
\tilde{f}^{2}(\tau)=\left(\frac{b}{a}\right)^{2} \tau+c \text {. }
$$

ou seja, $\tilde{f}^{2}$ é afim. 
ii) Vamos supor que $\forall z \neq 0 z \neq 0, f(\mathcal{O}(z)) \cap \mathcal{O}(z)=\emptyset$. Tomemos neste caso $\Sigma=S^{1}$. Como $D f(0)$ não tem autovalores reais (pois se $D f(0)$ tivesse autovalores reais, haveriam subespaços de $\mathbb{R}^{2}$ invariantes por $D f(0)$; como as órbitas de $X$ são raios, então haveriam órbitas de $X$ invariantes por $D f(0)$, o que não acontece) temos que $D f(0)=A=\left(\begin{array}{rr}a & b \\ -b & a\end{array}\right)$. Dessa forma,

$$
f(x)=A x+R(x)
$$

onde $x=\left(x_{1}, x_{2}\right)$ e $\lim _{x \rightarrow 0} \frac{R(x)}{\|x\|}=0 . \quad$ Seja $\tau=(\cos \theta, \operatorname{sen} \theta) \in \Sigma$. Temos $X_{t}(\tau)=e^{\alpha t}(\cos \theta, \operatorname{sen} \theta)$. Seja $r=\left\|X_{t}(\tau)\right\|=e^{\alpha t}$. Substituindo em $X_{t}(\tau)$, conseguimos uma expressão para a órbita do campo $X$ em função de $r$ :

$$
X_{r}(\tau)=r(\cos \theta, \operatorname{sen} \theta)=r \tau
$$

De 3.5 temos

$$
f\left(X_{r}(\tau)\right)=A r r+R\left(X_{r}(\tau)\right)
$$

Por outro lado, como $f$ manda a órbita de $X$ pelo ponto $\tau$ na órbita pelo ponto $\tilde{f}(\tau)$, temos de 3.6 que obtemos

$$
f\left(X_{r}(\tau)\right)=\left(f_{1}\left(X_{r}(\tau)\right), f_{2}\left(X_{r}(\tau)\right)=\|\left(f_{1}\left(X_{r}(\tau)\right), f_{2}\left(X_{r}(\tau)\right) \| \tilde{f}(\tau) .\right.\right.
$$

Como $\|\left(f_{1}\left(X_{r}(\tau)\right), f_{2}\left(X_{r}(\tau)\right) \|=\sqrt{a^{2}+b^{2}} r+\bar{R}\left(X_{r}(\tau)\right)\right.$, onde $\lim _{x \rightarrow 0} \frac{\bar{R}(x)}{\|x\|}=0$,

$$
f\left(X_{r}(\tau)\right)=\left[\sqrt{a^{2}+b^{2}} r+\bar{R}\left(X_{r}(\tau)\right)\right] \tilde{f}(\tau) .
$$

Comparando esta última expressão com 3.7 , temos

$$
A r \tau+R\left(X_{r}(\tau)\right)=\left[\sqrt{a^{2}+b^{2}} r+\bar{R}\left(X_{r}(\tau)\right)\right] \tilde{f}(\tau) .
$$

Multiplicando por $r^{-1}$ segue que

$$
A \tau+r^{-1} R\left(X_{r}(\tau)\right)=\left[\sqrt{a^{2}+b^{2}}+r^{-1} R\left(X_{r}(\tau)\right)\right] \tilde{f}(\tau) .
$$


Fazendo $r \rightarrow 0$ obtemos:

$$
\tilde{f}(\tau)=\left(\sqrt{a^{2}+b^{2}}\right)^{-1} A \tau
$$

ou seja, $\tilde{f}$ é uma rotação.

CASO B: Supondo $\operatorname{dim} E=1$, as órbitas de $X$ são como as da Figura 3.5, sendo $X=\left(\begin{array}{cc}\alpha & 1 \\ 0 & \alpha\end{array}\right)$.

Seja $\{v, z\}$ base para $\mathbb{R}^{2}$, onde $v \in E$. Dado $x \in \mathbb{R}^{2}$, sejam $\left(x_{1}, x_{2}\right)$ as coordenadas de $x$ com relação a base $\{v, z\}$. Como $D X(0)$ e $D f(0)$ comutam, temos que $D f(0)=\left(\begin{array}{ll}a & b \\ 0 & a\end{array}\right)$, onde $a=\frac{\partial f_{1}}{\partial x_{1}}(0)$ e $b=\frac{\partial f_{1}}{\partial x_{2}}(0)$. Logo, $f=\left(f_{1}, f_{2}\right)$ é dada por:

$$
f_{1}(x)=a x_{1}+b x_{2}+R_{1}(x) \text { e } f_{2}(x)=a x_{2}+R_{2}(x),
$$

onde $\lim _{x \rightarrow 0} \frac{R_{j}(x)}{\|x\|}=0, j=1,2$.

Vamos supor $a>0$. Então os subespaços $H_{+}=\left\{\left(x_{1}, x_{2}\right) \in \mathbb{R}^{2} ; \quad x_{2}>0\right\} \mathrm{e}$ $H_{-}=\left\{\left(x_{1}, x_{2}\right) \in \mathbb{R}^{2} ; \quad x_{2}<0\right\}$ são invariantes por $f$. Resulta da continuidade de - $f$ que $E$ é invariante por $f$.

Tomemos $\Sigma=\Sigma_{1} \cup \Sigma_{-1}$, onde $\Sigma_{ \pm 1}=\left\{\left(x_{1}, \pm 1\right) ; \quad x_{1} \in E\right\}$. Observe que $\Sigma$ está bem definido visto que toda órbita de $X$ em $H_{+}$intercepta a reta $\Sigma_{1}$ em um único ponto (análogo para uma órbita em $H_{-}$).

Seja $(\tau, 1) \in \Sigma$. Denotaremos às vezes este ponto simplesmente por $\tau$. A órbita de $X$ por $(\tau, 1)$ é dada por $X_{t}(\tau)=e^{\alpha t}(\tau+t, 1)$. Fazendo $u=e^{\alpha t}$ obtemos $t=\frac{1}{\alpha} \ln u$. Substituindo em $X_{t}(\tau)$, obtemos uma expressão para a órbita de $X$ em função da segunda coordenada:

$$
X_{u}(\tau)=\left(u\left(\tau+\frac{1}{\alpha} \ln u\right), u\right)
$$




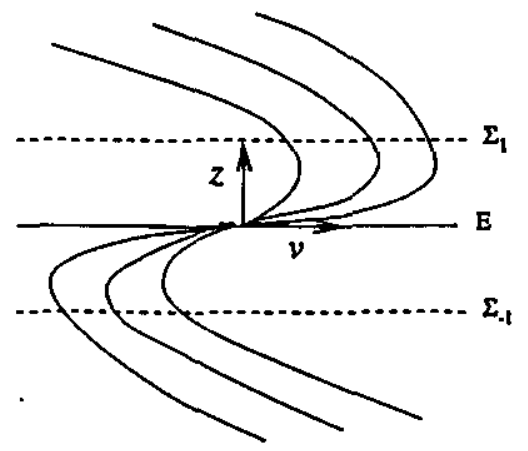

Figura 3.5: As órbitas de $X$ para o caso em que seus autovalores são iguais $\left(\alpha_{1}=\alpha_{2}=\alpha\right)$ e $\operatorname{dim} E=1$, sendo $E$ o autoespaço associado a $\alpha . \Sigma=\Sigma_{1} \cup \Sigma_{-1}$.

De 3.8 segue que

$$
f\left(X_{u}(\tau)\right)=\left(a u\left(\tau+\frac{1}{\alpha} \ln u\right)+b u+R_{1}\left(X_{u}(\tau)\right), a u+R_{2}\left(X_{u}(\tau)\right)\right) .
$$

Como $f$ manda a órbita de $X$ pelo ponto $\tau$ na órbita pelo ponto $\tilde{f}(\tau)$, temos de 3.9 que

$$
f\left(X_{u}(\tau)\right)=\left(\left[a u+R_{2}\left(X_{u}(\tau)\right)\right]\left[\tilde{f}(\tau)+\frac{1}{\alpha} \ln \left[a u+R_{2}\left(X_{u}(\tau)\right)\right]\right], a u+R_{2}\left(X_{u}(\tau)\right)\right)
$$

Comparando as duas últimas expressões temos

$$
\begin{aligned}
a u\left(\tau+\frac{1}{\alpha} \ln u\right)+b u+R_{1}\left(X_{u}(\tau)\right) & = \\
= & {\left[a u+R_{2}\left(X_{u}(\tau)\right)\right]\left[\tilde{f}(\tau)+\frac{1}{\alpha} \ln \left[a u+R_{2}\left(X_{u}(\tau)\right)\right]\right] }
\end{aligned}
$$

Multiplicando por $u^{-1}$ segue que

$$
\begin{aligned}
a\left(\tau+\frac{1}{\alpha} \ln u\right)+b+u^{-1} & R_{1}\left(X_{u}(\tau)\right)= \\
= & {\left[a u+R_{2}\left(X_{u}(\tau)\right)\right]\left[\frac{\tilde{f}(\tau)}{u}+\frac{u^{-1}}{\alpha} \ln \left[a u+R_{2}\left(X_{u}(\tau)\right)\right]\right] . }
\end{aligned}
$$


Derivando com relação a $\tau$ obtemos

$$
\begin{aligned}
& a+D_{1} R_{1}\left(X_{u}(\tau)\right)=u D_{1} R_{2}\left(X_{u}(\tau)\right)\left[\frac{\tilde{f}(\tau)}{u}+\frac{u^{-1}}{\alpha} \ln \left[a u+R_{2}\left(X_{u}(\tau)\right)\right]+\right. \\
&+\left[a u+R_{2}\left(X_{u}(\tau)\right)\right]\left[\frac{\tilde{f}^{\prime}(\tau)}{u}+\frac{1}{\alpha\left[a u+R_{2}\left(X_{u}(\tau)\right)\right]} D_{1} R_{2}\left(X_{u}(\tau)\right)\right]
\end{aligned}
$$

ou ainda,

$$
\begin{array}{r}
a+D_{1} R_{1}\left(X_{u}(\tau)\right)=\tilde{f}(\tau) D_{1} R_{2}\left(X_{u}(\tau)\right)+\frac{1}{\alpha} D_{1} R_{2}\left(X_{u}(\tau)\right) u \frac{\ln \left(a u+R_{2}\left(X_{u}(\tau)\right)\right)}{u}+ \\
+a \tilde{f}^{\prime}(\tau)+\tilde{f}^{\prime}(\tau) \frac{R_{2}\left(X_{u}(\tau)\right)}{u}+\frac{D_{1} R_{2}\left(X_{u}(\tau)\right)}{\alpha}
\end{array}
$$

Faremos $u \rightarrow 0$ nesta última expressão. Temos que

- $\lim _{u \rightarrow 0} D_{1} R_{j}\left(X_{u}(\tau)\right)=0 ; j=1,2$;

- $\lim _{u \rightarrow 0} \frac{R_{2}\left(X_{u}(\tau)\right)}{u}=0 \mathrm{e}$

- $\lim _{u \rightarrow 0}\left(u \cdot \frac{\ln \left(a u+R_{2}\left(X_{u}(\tau)\right)\right.}{u}\right)=0$ (pois $\frac{\ln \left[a u+R_{2}\left(X_{u}(\tau)\right)\right]}{u}$ é limitado visto que $\ln x<x)$.

Observe que apenas o limite $\lim _{u \rightarrow 0} \frac{R_{2}\left(X_{u}(\tau)\right)}{u}=0$ não é trivial. Iremos prová-lo pois foi deste limite que surgiu a necessidade de impormos que $f \in C^{2}$.

Mostremos então que $\lim _{u \rightarrow 0} \frac{R_{2}\left(X_{u}(\tau)\right)}{u}=0$. Como $f \in C^{2}$ temos que, para $x=$ $\left(x_{1}, x_{2}\right)$,

$$
f_{2}\left(x_{1}, x_{2}\right)=a x_{2}+\frac{\alpha}{2} x_{1}^{2}+\frac{\beta}{2} x_{1} x_{2}+\frac{\gamma}{2} x_{2}^{2}+S_{2}\left(x_{1}, x_{2}\right)
$$

onde $\lim _{x \rightarrow 0} \frac{S_{2}(x)}{\|x\|^{2}}$

Como $E$ é invariante por $f, f_{2}\left(x_{1}, 0\right)=0$. Logo $\frac{\alpha}{2} x_{1}^{2}=0 . \quad$ Assim, $R_{2}(x)=\frac{\beta}{2} x_{1} x_{2}+\frac{\gamma}{2} x_{2}^{2}+S_{2}\left(x_{1}, x_{2}\right)$. Portanto,

$$
R_{2}\left(X_{u}(\tau)\right)=\frac{\beta}{2} u^{2}\left(\tau+\frac{1}{\alpha} \ln u\right)+\frac{\gamma}{2} u^{2}+S_{2}\left(X_{u}(\tau)\right)
$$


e assim,

$$
\lim _{u \rightarrow 0} \frac{R_{2}\left(X_{u}(\tau)\right)}{u}=0 \Longleftrightarrow \lim _{u \rightarrow 0} \frac{S_{2}\left(X_{u}(\tau)\right)}{u}=0
$$

Mas este último limite é verdadeiro pois $\frac{S_{2}\left(X_{u}(\tau)\right)}{u}=\frac{S_{2}\left(X_{u}(\tau)\right)}{\left\|X_{u}(\tau)\right\|^{2}} \cdot \frac{\left\|X_{u}(\tau)\right\|^{2}}{u} \mathrm{e}$ $\frac{\left\|X_{u}(\tau)\right\|^{2}}{u}$ é limitado quando $u \rightarrow 0$ (de fato,

$$
\frac{\left\|X_{u}(\tau)\right\|^{2}}{u}=\frac{u^{2}\left(\tau+\frac{1}{\alpha} \ln u\right)^{2}+u^{2}}{u}=u\left(\tau^{2}+2 \frac{\tau}{\alpha} \ln u+\frac{1}{\alpha^{2}} \ln ^{2} u+1\right)
$$

$u \ln ^{2} u=\frac{\ln ^{2} u}{\frac{1}{u}} \rightarrow 0$ por L'Hospital)

Resulta então que $a=a \tilde{f}^{\prime}(\tau)$, de onde segue que

$$
\tilde{f}(\tau)=\tau+c
$$

ou seja, $\tilde{f}$ é afim.

Se $a<0$ então, utilizando o mesmo raciocínio de casos anteriores, conclú́mos que

$$
\tilde{f}^{2}(\tau)=\tau+c
$$

ou seja, $\tilde{f}^{2}$ é afim, como queríamos. Isto completa a prova desta proposição.

Tendo provado a proposição acima, podemos voltar a análise da estabilidade estrutural de $\bar{f}: S^{1} \rightarrow S^{1}$. No caso $A$ (ii), em que $\bar{f}$ é uma rotação, temos que $\vec{f}$ não é estruturalmente estável. Resta-nos analisar o caso A (i) e o caso B. Vamos analisá-los separadamente:

- Caso A (i). Como $L$ é invariante por $f$, temos que $y^{ \pm}=(0, \pm 1)$ são pontos fixos de $\bar{f}$. Pela Observação 3.2 do Lema 3.2 temos que estes pontos serão hiperbólicos $\Leftrightarrow \alpha_{2} \ln |a| \neq \alpha_{1} \ln |b| \Leftrightarrow|a| \neq|b|$. Como $\tilde{f}(\tau)=\frac{b}{a} \tau+c, \tau \in \Sigma$, 
nos que se $|a| \neq|b|$ então $\vec{f}$ tem mais dois pontos periódicos, de período 1 u 2 conforme $a>0$ ou $a<0$, respectivamente, os quais denotaremos por $x^{ \pm}$ e que também são hiperbólicos visto que $\tilde{f}^{\prime}\left(x^{ \pm}\right)=\frac{b}{a}\left(\mathrm{e}\left(\tilde{f}^{2}\right)^{\prime}\left(x^{ \pm}\right)=\frac{b^{2}}{a^{2}}\right)$. Com o mesmo raciocínio anterior, este são os únicos pontos pertencentes a $\Omega(\bar{f})$. Portanto, $\bar{f}$ é estruturalmente estável $\Leftrightarrow|a| \neq|b| \Leftrightarrow \alpha_{2} \ln |a| \neq \alpha_{1} \ln |b|$.

- Caso B. Como $E$ é invariante por $f$ temos que $x^{ \pm}=( \pm 1,0)$ são pontos periódicos de $\bar{f}$ de período 1 ou 2 . Sendo $\tilde{f}(\tau)=\tau+c$ temos que ou todos os pontos de $S^{1}$ são periódicos de $\bar{f}$ (caso em que $c=0$ ) e daí $\bar{f}$ não é estruturalmente estável, ou então $x^{ \pm}$são os únicos pontos periódicos de $\bar{f}$. Cálculos análogos aos anteriores nos mostram que $x^{ \pm}$não são pontos hiperbólicos de $\bar{f}$ e portanto $\bar{f}$ não é estruturalmente estável.

\subsection{Observação}

Sintetizamos os resultados das seções precedentes com o seguinte teorema:

Teorema 3.1 Sejam $X$ um campo de vetores linear hiperbólico de $\mathbb{R}^{2}, U$ um aberto de $\mathbb{R}^{2}$ contendo a origem ef $U \rightarrow \mathbb{R}^{2}$ um difeomorfismo local que aplica órbitas de $X$ em $U$ em órbitas de $X$ em $\mathbb{R}^{2}$, preservando a orientação. Sejam $\alpha$ e $\beta$ os autovalores de $X$ e a e b os correspondentes de $D f(0)$. Então $\bar{f}$ tem um número finito de pontos periódicos, todos eles hiperbólicos, se e somente se $\alpha, \beta \in \mathbb{R} e$ $\alpha \ln |b| \neq \beta \ln |a|$. 


\section{Referências Bibliográficas}

[1] C. Camacho and A. Lins Neto, "Orbit Preserving Diffeomorphisms and the Stability of Lie Group Actions and Singular Foliations", Lectures Notes in Mathematics 597 Springer-Verlag (1976) 82-103.

[2] J. L. Arraut, "Circle Leaves of two Dimension Foliations" (Preprint).

[3] J. Palis Jr, W. de Melo, Introdução aos Sistemas Dinâmicos, Projeto Euclides - IMPA/CNPq (1977).

[4] J. Sotomayor, Liçôes de Equações Diferenciais Ordinárias, Projeto Euclides IMPA/CNPq (1979).

[5] Z. Nitecki, Differentiable Dynamics, M.I.T. Press (1971).

[6] D. K. Arrowsmith and C. M. Place, An Introduction to Dynamical Systems, Cambridge University Press (1990). 